\title{
Vibrational spectra of acid and alkaline glycine salts
}

\author{
Mário T. Rosado ${ }^{a}$, Maria Leonor T.S. Duarte ${ }^{a}$, Rui Fausto ${ }^{\text {b,* }}$ \\ ${ }^{a}$ Departamento de Química e Bioquímica, Faculdade de Ciências da Universidade de Lisboa, Ed. C1-5, Campo Grande, 1700 Lisbon, \\ Portugal \\ b Departamento de Química, Universidade de Coimbra, 3049 Coimbra, Portugal
}

Received 5 September 1997; accepted 5 December 1997

\begin{abstract}
The infrared and Raman spectra of crystalline acid $\left({ }^{+} \mathrm{NH}_{3} \mathrm{CH}_{2} \mathrm{COOH} \cdot \mathrm{Cl}^{-}\right)$and alkaline $\left(\mathrm{Na}^{+} \cdot \mathrm{NH}_{2} \mathrm{CH}_{2} \mathrm{COO}^{-}\right)$ glycine salts were recorded and interpreted. The assignments were confirmed by comparison with the infrared and Raman spectra of crystalline glycine (zwitterion, ${ }^{+} \mathrm{NH}_{3} \mathrm{CH}_{2} \mathrm{COO}^{-}$) and the infrared spectrum of this molecule isolated in an argon matrix at low temperature $(10 \mathrm{~K})$ (neutral form, $\mathrm{NH}_{2} \mathrm{CH}_{2} \mathrm{COOH}$ ). Further insight on the features of the vibrational spectra of these substances was achieved by ab initio $\left(\mathrm{HF} / 6-31 \mathrm{G}^{*}\right)$ frequency and intensity calculations and potential energy distribution calculations resulting from normal coordinate analysis. (C) 1998 Elsevier Science B.V.
\end{abstract}

Keywords: Glycine (neutral and zwitterionic forms); Acid and alkaline glycine salts; Vibrational (IR and Raman) spectra; Ab initio prediction of vibrational spectra

\section{Introduction}

Glycine is the simplest protein structural unit and the smallest amino acid that can act as neuroinhibitor in mammalian central nervous systems [1,2]. Since vibrational spectroscopy can provide the key to elucidation of both structural and dynamical properties and can be used to establish fundamental correlations between these and the chemical or biochemical behaviour of these substances, considerable effort has been dedicated to such studies on glycine under various experimental conditions [2-12]. Interesting vibrational features of the infrared spectra of glycine, both in the zwitterionic [2-9] and neutral forms

\footnotetext{
* Corresponding author. Tel.: + 351-39-22826; fax: + 351-3927703; e-mail: rfausto@gemini.ci.uc.pt.
}

$[10,11]$, have previously been studied, although the main focus has been on the zwitterion, due to its greater biological importance.

The assignment of bands in the infrared spectrum of crystalline glycine (zwitterion) presents difficulties, due to the presence of some regions with very broad and structured bands (e.g. $\nu \mathrm{OH}, \nu \mathrm{NH}$ and $\nu \mathrm{CH}$ stretching regions). The study of the vibrational spectra of glycine alkaline and acid salts appeared to be a promising way of understanding complex features of the zwitterion spectra, since $-\mathrm{NH}_{2},-\mathrm{COO}^{-}$ and $-\mathrm{COOH},-\mathrm{NH}_{3}^{+}$groups are present in alkaline and acid salts, respectively. However, glycine salts have not previously received much attention.

Williams et al. tried to correlate vibrational spectra of the salts with ab initio calculations [12]. However, these focused only on spectra in aqueous solu- 
tion and the calculations were performed with a relatively modest basis set (4-31G).

In this work, FT-IR and Raman spectra of crystalline glycine salts were assigned with the help of polarized wavefunction double- $\zeta \mathrm{HF} / 6-31 \mathrm{G}^{*} \mathrm{ab}$ initio vibrational frequency calculations and normal coordinate analysis. All calculations were applied to the most stable conformer of each molecule studied (Fig. 1) and, since the present studies used the crystalline compounds, the cyclic dimer of the acid salt (see Fig. 1) was also considered.

The assignment of vibrational spectra was helped by extensive comparison with the spectra of zwitterionic and neutral glycine. The zwitterionic form of glycine constitutes a unique situation, because it does not correspond to a minimum in the potential energy surface of the isolated molecule [13]. The calculations were carried out using the basic molecular geometry previously determined by neutron diffraction for an individual molecule in the $\alpha$-crystalline

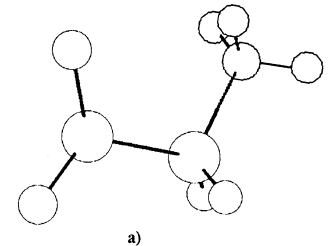

a)

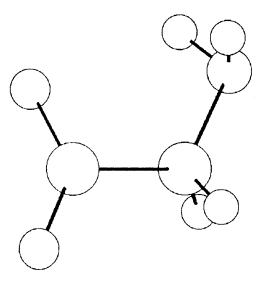

c)

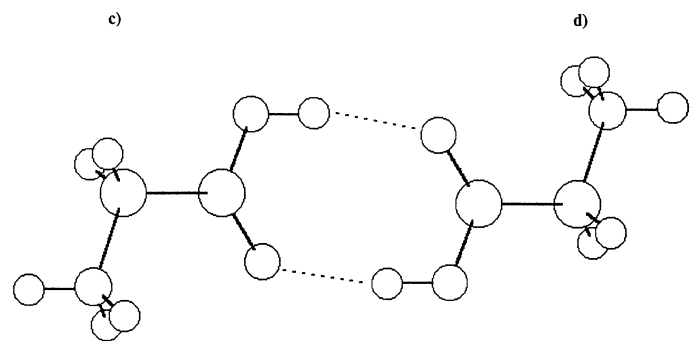

e)

Fig. 1. Molecular structures of the various systems studied: (a) zwitterionic glycine; (b) neutral glycine; (c) glycine alkaline salt; (d) glycine acid salt (monomer) and (e) glycine acid salt (dimer). modification of this compound [14] (glycine crystallizes in three different modifications, the $\alpha, \beta$ and $\gamma$ forms [2], the $\alpha$-form corresponding to the stable modification at room temperature). For zwitterionic glycine, the infrared and Raman spectra of the crystal ( $\alpha$-modification) were obtained, while the neutral form was studied by matrix isolation infrared spectroscopy (MI-IR), in Argon, at $10 \mathrm{~K}$.

\section{Experimental and computational methods}

$\alpha$-Glycine p.a. $+98 \%$ was obtained from Merck. The alkaline salt, the glycinate $\left(\mathrm{Na}^{+}\right.$. $\mathrm{NH}_{2} \mathrm{CH}_{2} \mathrm{COO}^{-}$), was prepared by dissolving commercial glycine p.a. in a saturated solution of $\mathrm{NaOH}$. The solution $(\mathrm{pH}>12)$ was dried in vacuum until complete solvent evaporation, and the crystals washed with methanol. The acid glycine salt $\left({ }^{+} \mathrm{NH}_{3} \mathrm{CH}_{2} \mathrm{COOH} \cdot \mathrm{Cl}^{-}\right)$was prepared by dissolution of the amino acid in concentrated $\mathrm{HCl}$ solution $(33 \%)$ and the resulting solvent $(\mathrm{pH}<3)$ was evaporated as for the alkaline salt.

Fourier transform infrared (FT-IR) spectra of crystalline materials as potassium bromide pellets were recorded with a Perkin Elmer PE 1760 FT-IR spectrometer, in the $4000-400 \mathrm{~cm}^{-1}$ region, with a resolution of $2 \mathrm{~cm}^{-1}$.

Raman spectra were obtained using a SPEX 1403 double monochromator spectrometer (focal distance $0.85 \mathrm{~m}$, aperture $f / 7.8$ ), equipped with holographic gratings with 1800 grooves $\mathrm{mm}^{-1}$ (Ref. 18001SHD). The $514.5 \mathrm{~nm}$ argon laser (Spectra-Physics, model 164-05) line, adjusted to provide $220 \mathrm{~mW}$ power at the sample, was used as excitation radiation. Detection was effected using a thermoelectrically cooled Hamamatsu R928 photomultiplier. Spectra were recorded using increments of $1 \mathrm{~cm}^{-1}$ and integration times of $1 \mathrm{~s}$. Under these conditions, the estimated errors in wavenumbers are $\pm 1 \mathrm{~cm}^{-1}$.

The matrix isolation spectrum (MI-IR) of the neutral form of glycine was recorded with a dispersive Specord IR75 (updated) infrared spectrometer and a resolution of $0.2 \mathrm{~cm}^{-1}$. The complete description of the set-up used for these experiments is described elsewhere [10].

$\mathrm{Ab}$ initio calculations were performed with a PC equipped with a Am486DX4/100 MHz processor, 
using Gaussian 92 for Windows/Revision G-3 [15]. These calculations were executed at the HartreeFock level of theory with the $6-31 \mathrm{G}^{*}$ basis set [16]. Molecular geometries were fully optimized by the force gradient method using Berny's algorithm [17]. The largest residual coordinate forces were always less than $3 \times 10^{-4}$ hartree bohr $^{-1}$ (1 hartree $=$ $\left.2625.5001 \mathrm{~kJ} \mathrm{~mol}^{-1} ; 1 \mathrm{bohr}=5.29177 \times 10^{-11} \mathrm{~m}\right)$ or hartree $\mathrm{rad}^{-1}$, for bond stretches and angle bends, respectively. The stopping criterion for the SCF iterative process required a density matrix convergence of less than $10^{-8}$.

The force constants (symmetry internal coordinates) used in the normal coordinate analysis were obtained from the ab initio Cartesian harmonic force constants using the program TRANSFORMER (version 2.0) [18]. This program was also used to prepare input data for the normal coordinate analysis programs: BUILD-G and VIBRAT (Refs. [19] ${ }^{1}$ and [20]), which enabled the calculation of the potential energy distribution (PED) of each of the normal modes of vibration.

The ab initio calculated force fields were scaled down using a single scale factor (0.89), which is accepted to be the best for the Hartree-Fock level of calculations [21]. This very simple scaling preserves the potential energy distributions as they emerge from the ab initio calculation and has an important advantage over more elaborate force field scaling procedures that use more than one scale factor and usually gives rise to marked PED distortions from the $a b$ initio calculated values.

\section{Results and discussion}

The optimized molecular geometries for the lowest energy conformation of the various molecules studied are shown in Table 1. The vibrational spectra of neutral and zwitterionic glycine (infrared and Raman) are shown in Figs. 2-4 and those of the alkaline and acid salts are presented in Figs. 5-8. Experimental and calculated vibrational frequencies,

\footnotetext{
${ }^{1}$ These programs incorporate several routines from programs GMAT and FPERT [19].
}

intensities, their potential energy distribution and proposed assignments are presented in Tables 2-5.

In general, the assignments made for glycine (both neutral and zwitterionic form) agree with those previously proposed [2-9] and, together with the results of the calculations, were used as a basis for assignment of the spectra of the two salts. The detailed analysis and discussion of the results will be divided into sections, corresponding approximately to different spectral regions.

\subsection{Region above $2000 \mathrm{~cm}^{-1}$}

Only neutral glycine and its acid salt contain the $\mathrm{OH}$ group. In the MI-IR spectrum of neutral glycine, as consequence of the isolated status in the $\mathrm{Ar}$ matrix, the $\mathrm{OH}$ stretching mode appears as a sharp band at $3560 \mathrm{~cm}^{-1}$. In the infrared spectrum of the acid salt, this appears as a broad band centered at $3439 \mathrm{~cm}^{-1}$. In this case, the band shape clearly shows the effect of association and hydrogen bonding. It is interesting to note that the calculated value for the frequency of this mode for a monomer in the acid salt calculations (3558 $\mathrm{cm}^{-1}$; see Table 5) is considerably higher than the experimental one, while the calculated value for the dimer $\left(3437 \mathrm{~cm}^{-1}\right.$; Table 6) is in good agreement.

Assignment of the $\mathrm{NH}$ and $\mathrm{CH}$ stretching modes in the neutral glycine infrared spectrum is straightforward, since these give rise to bands in a clear spectral region. In the alkaline salt, $\nu_{\mathrm{as}} \mathrm{CH}_{2}$ and $\nu_{\mathrm{s}} \mathrm{CH}_{2}$ are observed at 2945 and $2927 \mathrm{~cm}^{-1}$, in the infrared spectrum and give rise to strong Raman bands at 2941 and $2911 \mathrm{~cm}^{-1}$. Calculations on this molecule suggest the $\nu \mathrm{NH}_{2}$ bands should appear at lower frequencies than in isolated neutral glycine (see Tables 3 and 4). Thus, these modes are assigned to the two broad medium intensity bands at 3325 $\mathrm{cm}^{-1}\left(\nu_{\mathrm{as}} \mathrm{NH}_{2}\right)$ and $3212 \mathrm{~cm}^{-1}\left(\nu_{\mathrm{s}} \mathrm{NH}_{2}\right)$ in the Raman spectrum of this compound. In the corresponding infrared spectrum, the $\nu \mathrm{NH}_{2}$ vibrations give rise to the very broad feature from ca. 3000 to $3400 \mathrm{~cm}^{-1}$. The two infrared bands at 3439 and $3391 \mathrm{~cm}^{-1}$ are at too high frequencies for these modes and are attributed to the first overtone of the $\nu_{\mathrm{as}} \mathrm{COO}^{-}$stretching fundamental, which appears as a doublet at 1597 and $1561 \mathrm{~cm}^{-1}$. 
The infrared spectra of both crystalline glycine and its acid salt show a very broad and structured band spanning from 3400 to $2400 \mathrm{~cm}^{-1}$. This is typical of crystalline amino acids $[5,22]$ and has been ascribed to $\mathrm{NH}_{3}$ stretching modes, which sometimes obscure the $\mathrm{CH}_{2}$ stretching bands. In the Raman spectra, the $\nu \mathrm{CH}_{2}$ modes increase and the $\nu \mathrm{NH}_{3}$ modes decrease in intensity, which allows a clear observation of the first. The origin of the extensive broadening and complex structure of the $\nu \mathrm{NH}_{3}$ stretching region in the infrared spectrum of crystalline amino acids has been extensively discussed [5,23-26]. Although still controversial, it is generally considered that the observed band contour results essentially from Fermi resonance between the $\nu \mathrm{NH}_{3}$ stretching modes and combination bands involving mainly the $\delta \mathrm{NH}_{3}$ bending vibrations [5]. This interpretation is reinforced by the present results. Al-

Table 1

Calculated (ab initio 6-31G ${ }^{*}$ ) optimized molecular geometries ${ }^{\mathrm{a}}$

\begin{tabular}{|c|c|c|c|c|c|}
\hline Coordinate & Glycine (neutral form) & Glycine $^{\mathrm{b}}$ (zwitterion) & Alkaline salt & Acid salt (monomer) & Acid salt (dimer) \\
\hline $\mathrm{C}=\mathrm{O}$ & 1.188 & 1.233 & 1.233 & 1.184 & 1.197 \\
\hline $\mathrm{C}-\mathrm{C}$ & 1.515 & 1.553 & 1.553 & 1.517 & 1.515 \\
\hline $\mathrm{C}-\mathrm{O}$ & 1.330 & 1.237 & 1.237 & 1.301 & 1.289 \\
\hline $\mathrm{C}-\mathrm{N}$ & 1.439 & 1.496 & 1.463 & 1.496 & 1.495 \\
\hline $\mathrm{O}-\mathrm{H}$ & 0.952 & & & 0.957 & 0.964 \\
\hline \multirow[t]{2}{*}{$\mathrm{C}-\mathrm{H}$} & 1.085 & 1.080 & 1.086 & 1.080 & 1.081 \\
\hline & 1.085 & 1.080 & 1.089 & 1.080 & 1.081 \\
\hline \multirow{2}{*}{$\mathrm{N}-\mathrm{H}_{\mathrm{op}}$} & 1.002 & 1.012 & 1.005 & 1.012 & 1.013 \\
\hline & 1.002 & 1.012 & 1.005 & 1.012 & 1.013 \\
\hline $\mathrm{N}-\mathrm{H}_{\mathrm{ip}}$ & & 1.011 & & 1.011 & 1.013 \\
\hline $\mathrm{H} \ldots \mathrm{O}=$ & & & & & 1.948 \\
\hline $\mathrm{C}-\mathrm{C}-\mathrm{O}$ & 111.8 & 117.5 & 115.6 & 111.4 & 111.6 \\
\hline $\mathrm{C}-\mathrm{C}=\mathrm{O}$ & 125.4 & 117.1 & 116.7 & 120.8 & 121.1 \\
\hline $\mathrm{O}=\mathrm{C}-\mathrm{O}$ & 122.8 & 125.4 & 129.7 & 127.8 & 127.2 \\
\hline $\mathrm{N}-\mathrm{C}-\mathrm{C}$ & 115.0 & 111.9 & 115.7 & 106.8 & 108.6 \\
\hline $\mathrm{C}-\mathrm{O}-\mathrm{H}$ & 108.4 & & & 111.3 & 112.4 \\
\hline \multirow[t]{2}{*}{$\mathrm{C}-\mathrm{C}-\mathrm{H}$} & 107.7 & 108.8 & 109.0 & 111.4 & 110.7 \\
\hline & 107.7 & 110.5 & 107.4 & 111.4 & 110.7 \\
\hline \multirow[t]{2}{*}{$\mathrm{C}-\mathrm{N}-\mathrm{H}_{\mathrm{op}}$} & 110.4 & 110.4 & 104.6 & 110.9 & 111.6 \\
\hline & 110.4 & 111.7 & 106.9 & 110.9 & 111.6 \\
\hline $\mathrm{C}-\mathrm{N}-\mathrm{H}_{\mathrm{ip}}$ & & 112.1 & & 112.2 & 111.5 \\
\hline $\mathrm{O}-\mathrm{H} \ldots \mathrm{O}=$ & & & & & 170.3 \\
\hline $\mathrm{C}=\mathrm{O} \ldots \mathrm{H}$ & & & & & 130.1 \\
\hline $\mathrm{C}-\mathrm{C}(=\mathrm{O})-\mathrm{O}$ & 180.0 & 180.0 & 178.2 & 180.0 & 180.0 \\
\hline $\mathrm{N}-\mathrm{C}-\mathrm{C}=\mathrm{O}$ & 0.0 & 18.9 & 11.8 & 0.0 & 0.0 \\
\hline $\mathrm{O}=\mathrm{C}-\mathrm{O}-\mathrm{H}$ & 0.0 & & & 0.0 & 0.0 \\
\hline \multirow[t]{2}{*}{$\mathrm{H}-\mathrm{C}-\mathrm{C}=\mathrm{O}$} & -123.0 & -101.0 & -107.9 & -118.8 & -119.5 \\
\hline & 123.0 & 140.6 & 133.7 & 118.8 & 119.5 \\
\hline $\mathrm{H}_{\mathrm{op}}-\mathrm{N}-\mathrm{C}-\mathrm{C}$ & -58.6 & -58.1 & -37.7 & -58.5 & -59.3 \\
\hline $\mathrm{H}_{\mathrm{ip}}-\mathrm{N}-\mathrm{C}-\mathrm{C}$ & & -177.3 & & 180.0 & 180.0 \\
\hline
\end{tabular}

${ }^{a}$ Bond lengths in $\AA$, angles in degrees; geometries refer to the most stable conformer in the RHF/6-31G ${ }^{*}$ PES of each molecule studied. ${ }^{\mathrm{b}}$ Zwitterionic form of glycine does not correspond to a minimum in the PES of this molecule [13]. The geometry here presented and used to undertake the vibrational calculations was built as follows: (i) valence angles and dihedrals were taken from the neutron diffraction data relative to crystalline glycine ( $\alpha$-form) [14]; (ii) bond lengths were transferred from the $6-31 \mathrm{G}^{*}$ optimized structures here calculated for the alkaline salt $\left(\mathrm{COO}^{-}\right.$group) and acid salt $\left(\mathrm{NH}_{3}^{+}\right.$group). The calculated bond lengths obtained for the two salts were used instead of the experimental data obtained from neutron diffraction since otherwise it would not be possible to adopt the single force field scaling factor used in this study to fit the calculated frequencies to the experimental spectra also for the zwitterionic form of glycine. Indeed, the scale factor requires $6-31 \mathrm{G}^{*}$ consistent data, in particular in the case of bond lengths, whose particular values strongly influence the calculated frequencies. 

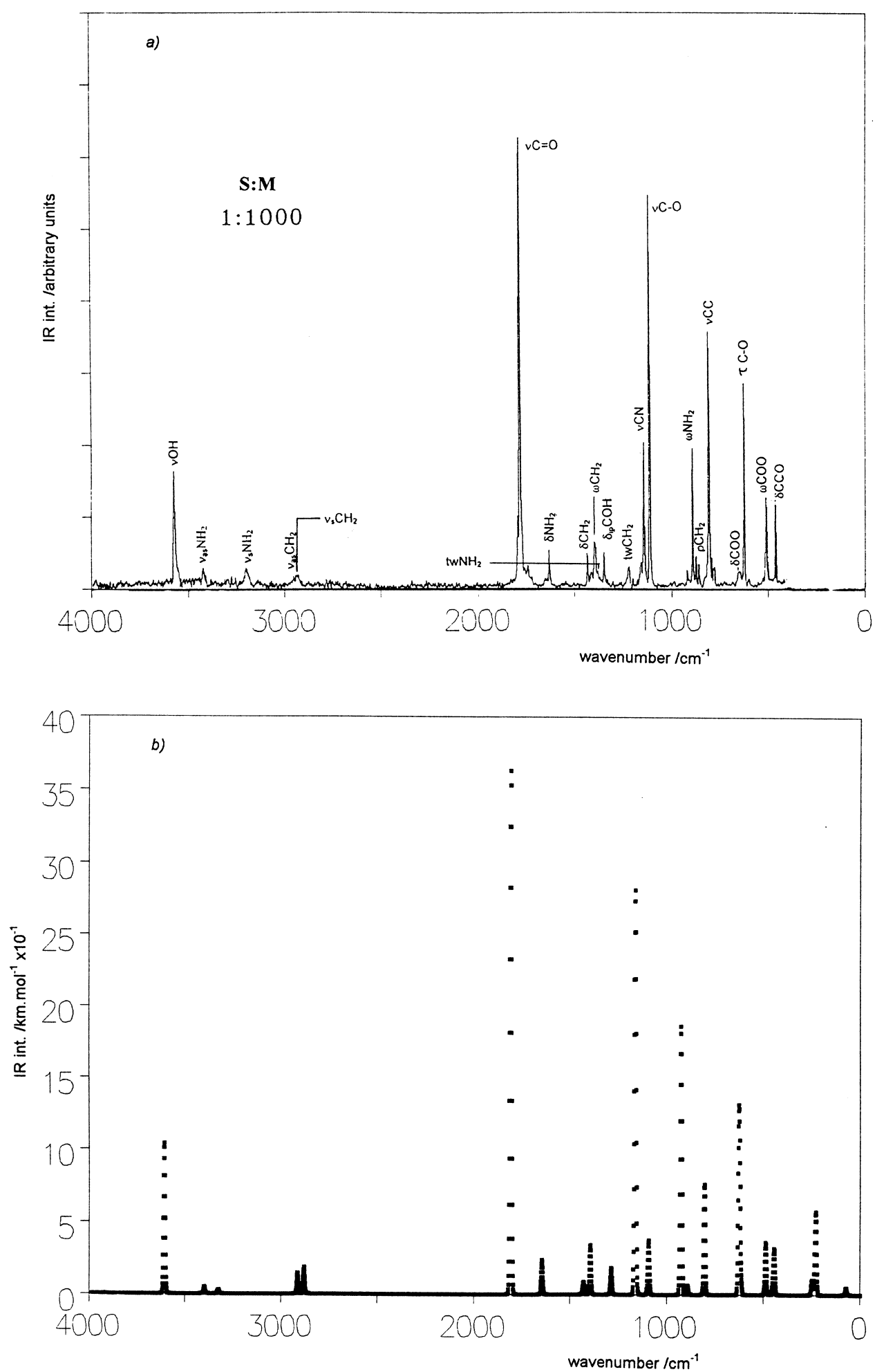

Fig. 2. Infrared spectra of neutral form of glycine: (a) experimental (MI-IR spectrum of glycine isolated in an Ar matrix at $10 \mathrm{~K}$ ) and (b) $6-31 \mathrm{G}^{*}$ predicted infrared spectrum (scaled). 

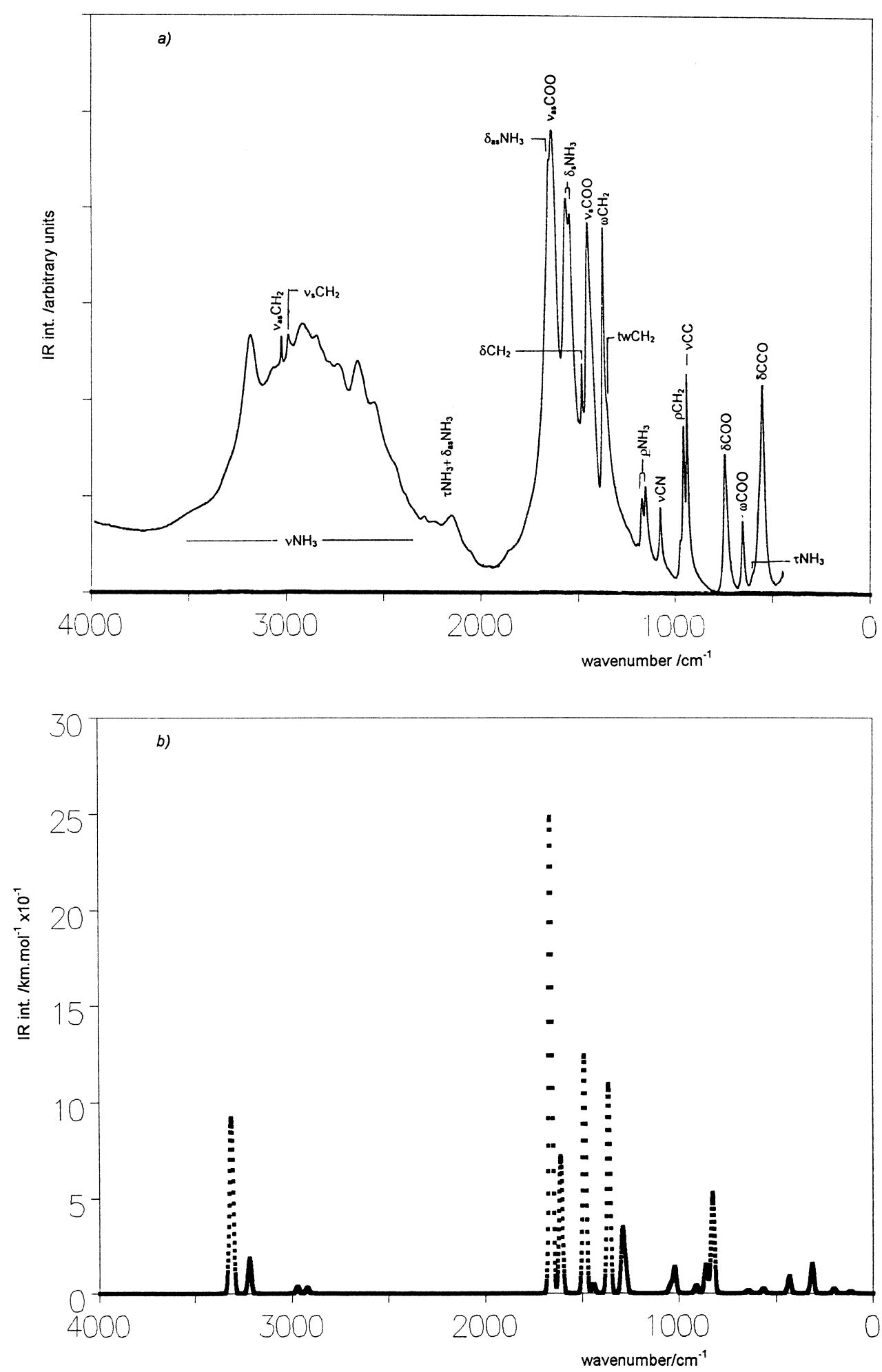

Fig. 3. Infrared spectra of zwitterionic glycine: (a) experimental (FT-IR spectrum of crystalline glycine at room temperature) and (b) $6-31 \mathrm{G}^{*}$ predicted infrared spectrum (scaled). 

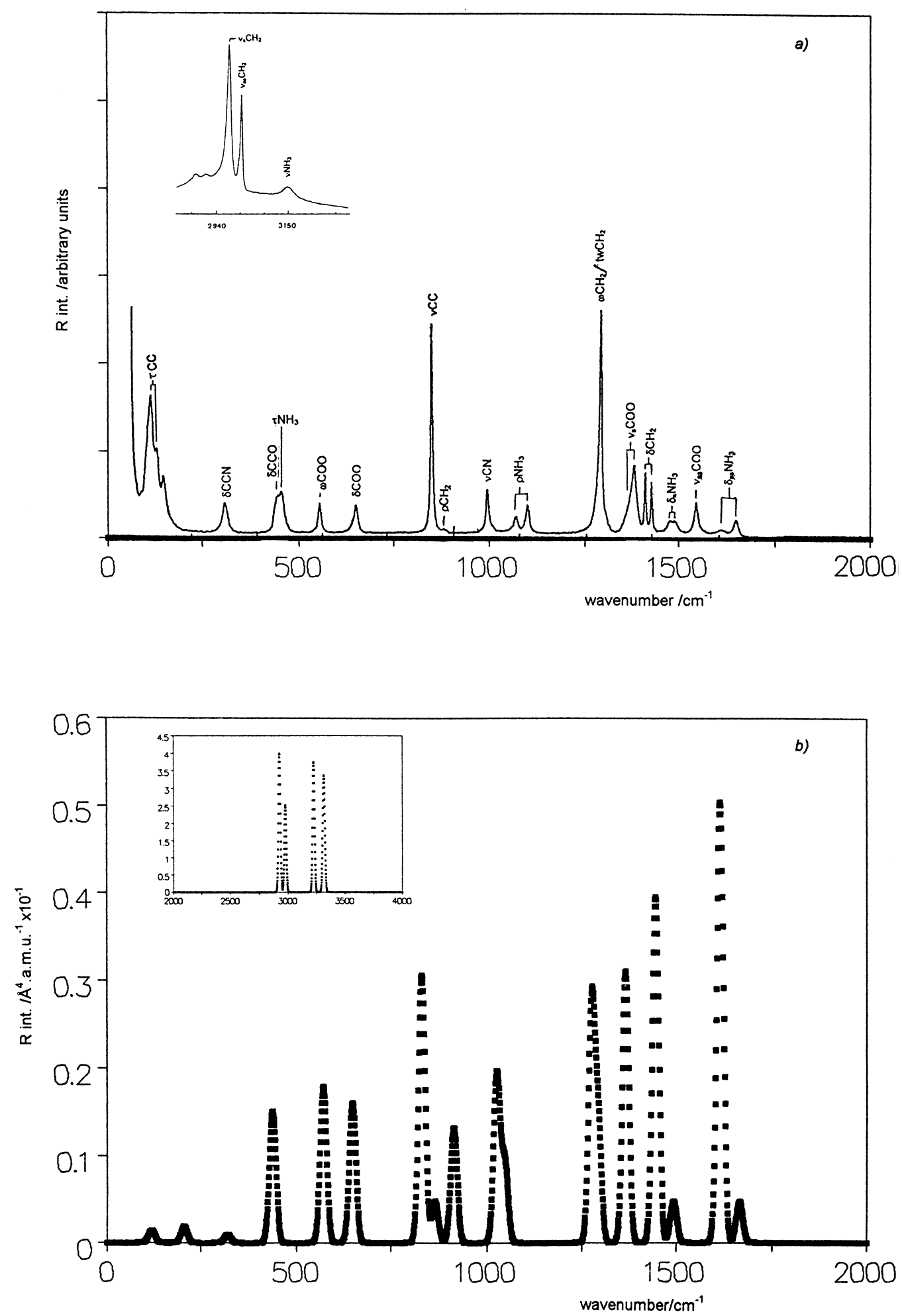

Fig. 4. Raman spectra of zwitterionic glycine: (a) experimental (Raman spectrum of crystalline glycine at room temperature) and (b) 6-31G* predicted Raman spectrum (scaled). 

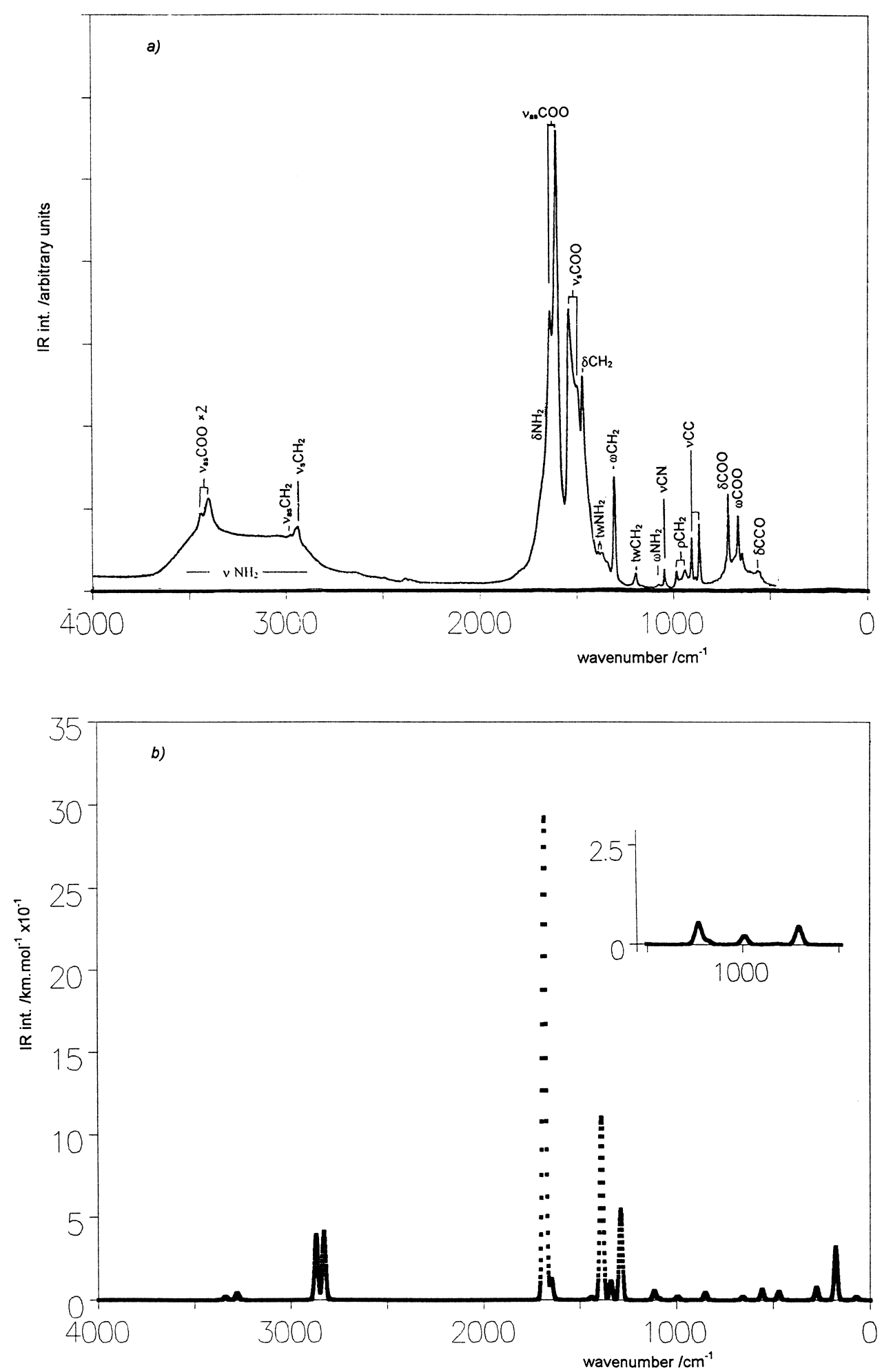

Fig. 5. Infrared spectra of glycine alkaline salt: (a) experimental (FT-IR spectrum of crystalline $\mathrm{Na}^{+} \cdot \mathrm{NH}_{2} \mathrm{CH}_{2} \mathrm{COO}^{-}$at room temperature) and (b) $6-31 \mathrm{G}^{*}$ predicted infrared spectrum (scaled). 

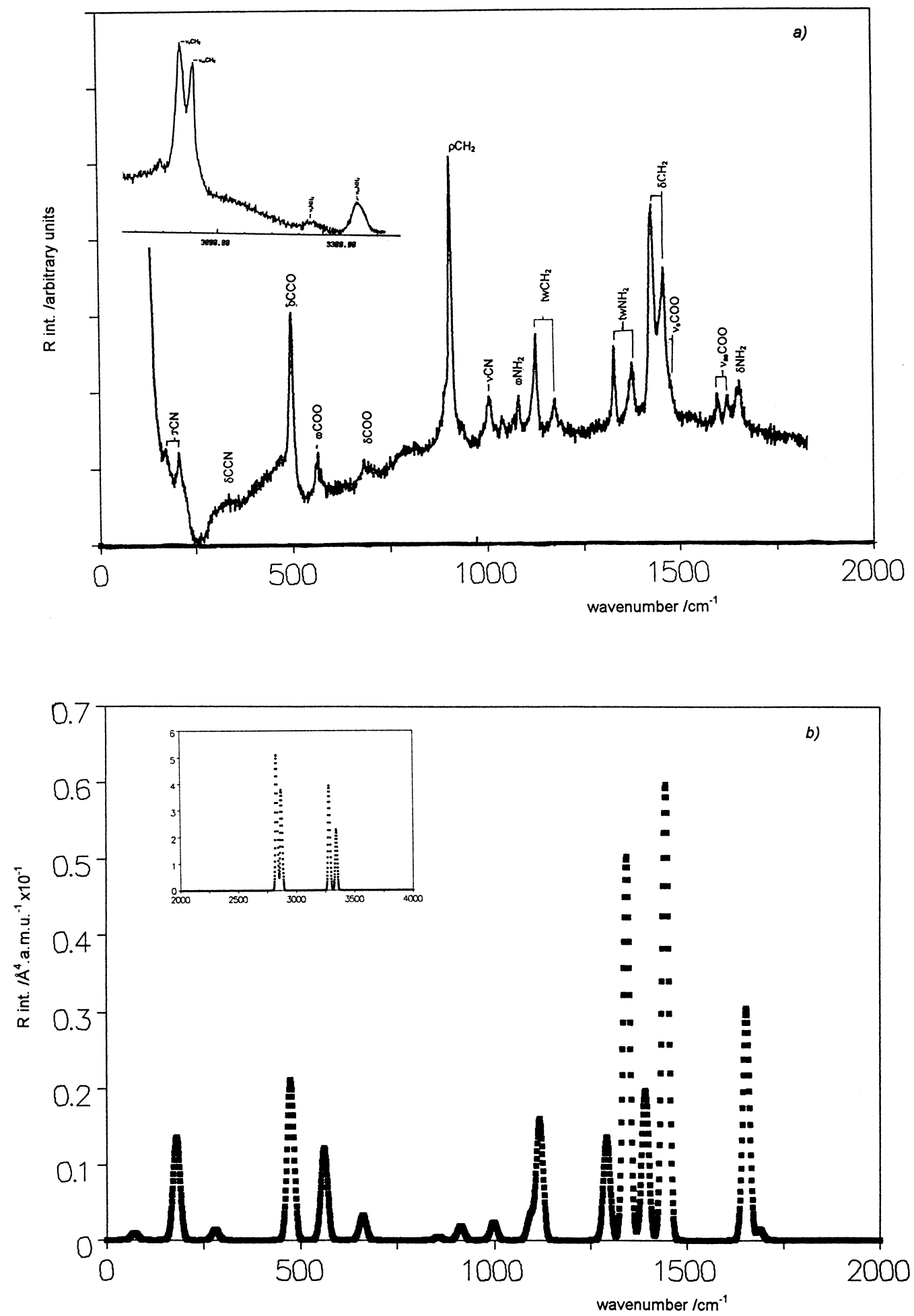

Fig. 6. Raman spectra of glycine alkaline salt: (a) experimental (Raman spectrum of crystalline $\mathrm{Na}^{+} \cdot \mathrm{NH}_{2} \mathrm{CH}_{2} \mathrm{COO}^{-}$at room temperature) and (b) $6-31 \mathrm{G}^{*}$ predicted Raman spectrum (scaled). 

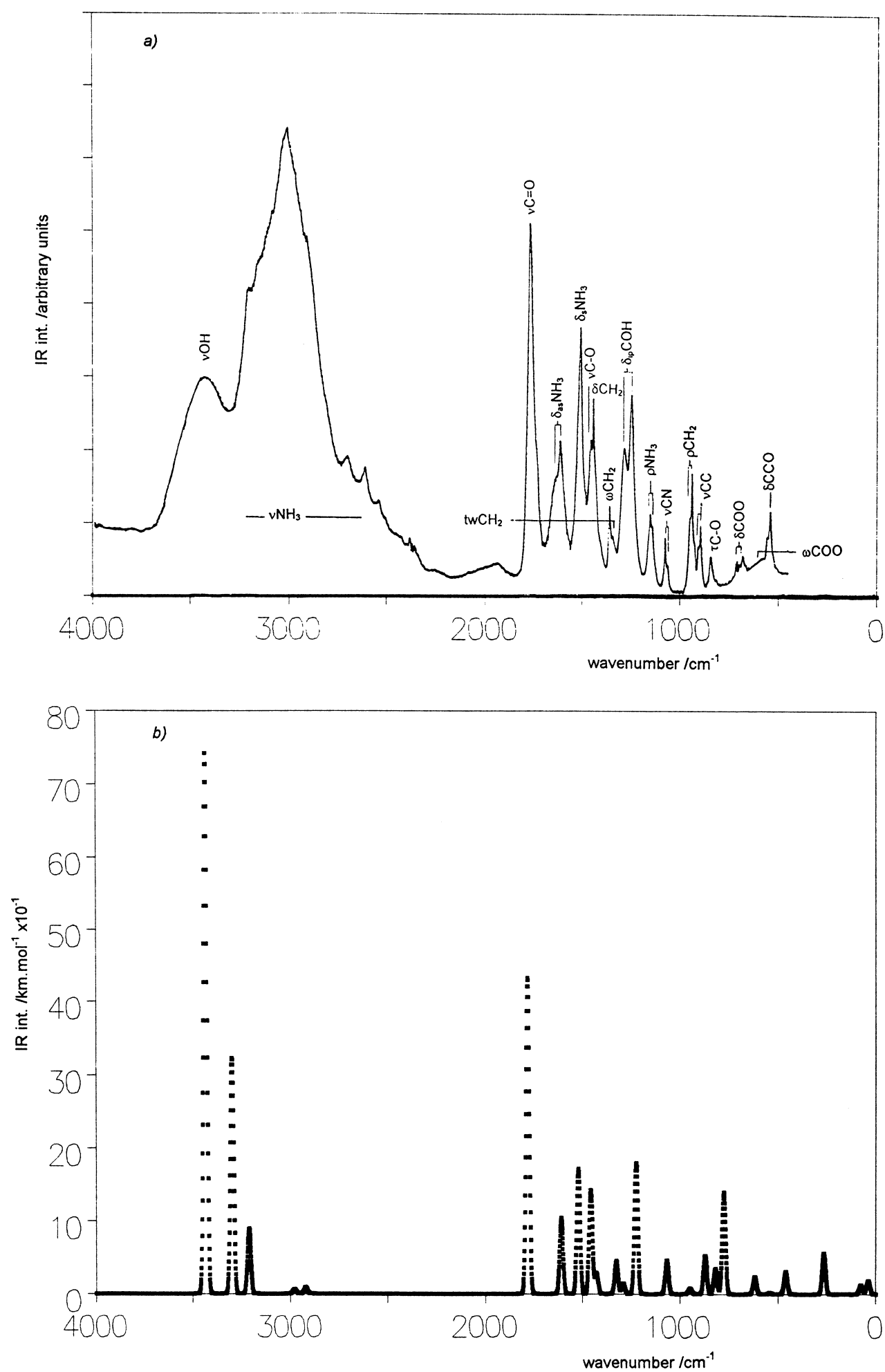

Fig. 7. Infrared spectra of glycine acid salt: (a) experimental (FT-IR spectrum of crystalline ${ }^{+} \mathrm{NH}_{3} \mathrm{CH}_{2} \mathrm{COOH} \cdot \mathrm{Cl}^{-}$at room temperature) and (b) $6-31 \mathrm{G}^{*}$ predicted infrared spectrum (dimer 'model'; scaled). 

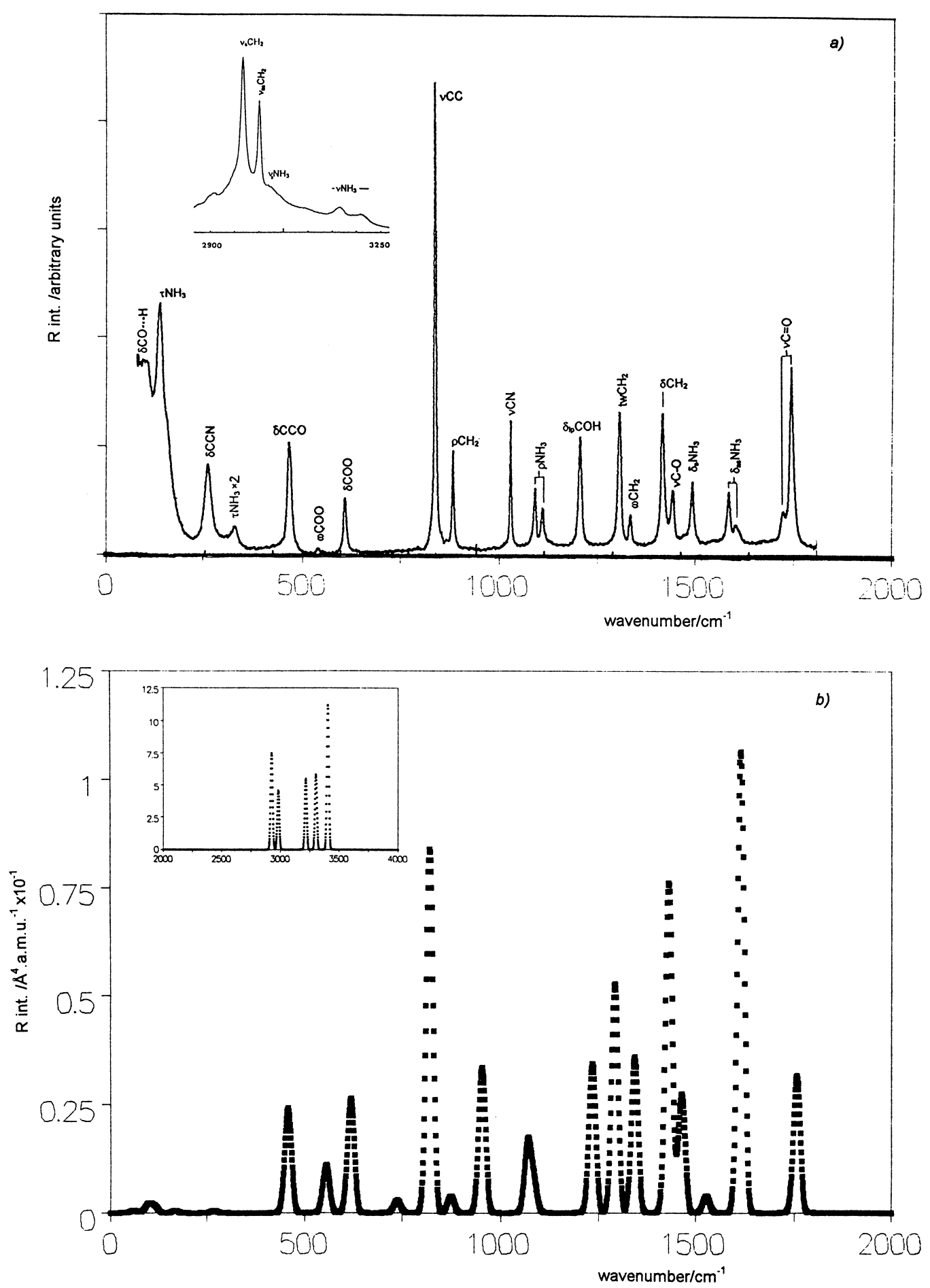

Fig. 8. Raman spectra of glycine alkaline salt: (a) experimental (Raman spectrum of crystalline ${ }^{+} \mathrm{NH}_{3} \mathrm{CH}_{2} \mathrm{COOH} \mathrm{Cl}^{-}$at room temperature) and (b) 6-31G* predicted Raman spectrum (dimer 'model'; scaled). 
Table 2

Spectra assignment and potential energy distribution for the zwitterionic form of glycine ${ }^{\mathrm{a}}$

\begin{tabular}{|c|c|c|c|c|c|c|c|c|c|}
\hline \multirow[t]{2}{*}{ Assignment } & \multirow[t]{2}{*}{ Symmetry } & \multicolumn{3}{|l|}{ Calculated } & \multicolumn{2}{|c|}{ Experimental (IR) } & \multicolumn{3}{|c|}{ Experimental (Raman) } \\
\hline & & Wavenumber & IR int. & $\mathrm{R}$ int. & Wavenumber & Intensity & Wavenumber & Intensity & P.E.D. \\
\hline$\nu_{\mathrm{as}} \mathrm{NH}_{3}$ & $A$ & 3319 & 131.0 & 27.8 & 3176,3040 & $\mathrm{~s}, \mathrm{~s}$ & 3149 & $\mathrm{~m}$ & $\nu_{\mathrm{as}}^{\prime} \mathrm{NH}_{3}$ \\
\hline$\nu_{\mathrm{as}}^{\mathrm{as}} \mathrm{NH}_{3}$ & $A$ & 3311 & 86.0 & 51.5 & 3176,3040 & $\mathrm{~s}, \mathrm{~s}$ & 3149 & $\mathrm{~m}$ & $\nu_{\mathrm{as}}^{\prime \prime} \mathrm{NH}_{3}$ \\
\hline$\nu_{\mathrm{s}} \mathrm{NH}_{3}$ & $A$ & 3224 & 38.9 & 79.5 & $2899^{\mathrm{b}}$ & $\mathrm{w}$ & 2911,2886 & $\mathrm{~m}, \mathrm{~m}$ & $\nu_{\mathrm{s}} \mathrm{NH}_{3}$ \\
\hline$\nu_{\mathrm{as}} \mathrm{CH}_{2}$ & $A$ & 2977 & 7.3 & 53.6 & 3007 & $\mathrm{w}$ & 3013 & $\mathrm{~s}$ & $\nu_{\mathrm{as}} \mathrm{CH}_{2}$ \\
\hline$\nu_{\mathrm{s}} \mathrm{CH}_{2}$ & $A$ & 2926 & 6.1 & 84.9 & 2970 & vs & 2978 & $\mathrm{~s}$ & $\nu_{\mathrm{s}} \mathrm{CH}_{2}$ \\
\hline$\nu_{\mathrm{as}} \mathrm{COO}$ & $A$ & 1666 & 528.5 & 1.0 & 1596 & vs & 1575 & w & $\nu_{\mathrm{as}} \mathrm{COO}(83), \delta_{\mathrm{as}}^{\prime} \mathrm{NH}_{3}(10)$ \\
\hline$\delta_{\mathrm{as}} \mathrm{NH}_{3}$ & $A$ & 1616 & 109.0 & 5.5 & 1615 & $\mathrm{~s}$ & 1676,1638 & $\mathrm{w}, \mathrm{vw}$ & $\delta_{\mathrm{as}}^{\prime} \mathrm{NH}_{3}(40), \delta_{\mathrm{as}}^{\prime \prime} \mathrm{NH}_{3}(40), \nu_{\mathrm{as}} \mathrm{COO}(10)$ \\
\hline$\delta_{\mathrm{as}} \mathrm{NH}_{3}$ & $A$ & 1614 & 44.3 & 5.3 & 1615 & s & 1676,1638 & $\mathrm{w}, \mathrm{vw}$ & $\delta_{\mathrm{as}}^{\prime \prime} \mathrm{NH}_{3}(47), \delta_{\mathrm{as}}^{\prime} \mathrm{NH}_{3}(42)$ \\
\hline$\delta_{\mathrm{s}} \mathrm{NH}_{3}$ & $A$ & 1493 & 264.2 & 1.0 & 1525,1505 & $\mathrm{~s}, \mathrm{~s}$ & 1522,1511 & $\mathrm{w}, \mathrm{w}$ & $\delta_{\mathrm{s}} \mathrm{NH}_{3}$ \\
\hline$\delta \mathrm{CH}_{2}$ & $A$ & 1446 & 9.7 & 8.4 & 1460,1444 & $\mathrm{vw}, \mathrm{m}$ & 1462,1446 & $\mathrm{~m}, \mathrm{~m}$ & $\delta \mathrm{CH}_{2}$ \\
\hline$\nu_{\mathrm{s}} \mathrm{COO}$ & $A$ & 1367 & 232.5 & 6.6 & 1413,1401 & $\mathrm{~s}, \mathrm{~m}$ & 1417,1404 & $\mathrm{~m}, \mathrm{sh}$ & $\nu_{\mathrm{s}} \mathrm{COO}(72), \nu \mathrm{CC}(20), \delta \mathrm{COO}(15), \omega \mathrm{CH}_{2}(14)$ \\
\hline$\omega \mathrm{CH}_{2}$ & $A$ & 1294 & 69.6 & 2.8 & 1334 & s & 1331 & $\mathrm{~s}$ & $\omega \mathrm{CH}_{2}(59), \nu_{\mathrm{s}} \mathrm{COO}(22)$ \\
\hline $\mathrm{twCH}_{2}$ & $A$ & 1277 & 26.6 & 5.8 & 1310 & $\mathrm{w}$ & 1331 & $\mathrm{~s}$ & $\mathrm{twCH}_{2}(46), \rho^{\prime \prime} \mathrm{NH}_{3}(18)$ \\
\hline$\rho \mathrm{NH}_{3}$ & $A$ & 1046 & 8.6 & 1.9 & 1131 & $\mathrm{w}$ & 1144 & w & $\begin{array}{l}\rho^{\prime} \mathrm{NH}_{3}(29), \mathrm{twCH}_{2}(21), \rho \mathrm{NH}_{3}(16), \omega \mathrm{COO}(15), \\
\rho \mathrm{CH}_{2}(11)\end{array}$ \\
\hline$\rho \mathrm{NH}_{3}$ & $A$ & 1025 & 28.3 & 4.1 & 1112 & $\mathrm{w}$ & 1114 & $\mathrm{w}$ & $\begin{array}{l}\rho^{\prime \prime} \mathrm{NH}_{3}(32), \mathrm{twCH}_{2}(19), \rho^{\prime} \mathrm{NH}_{3}(16), \rho \mathrm{CH}_{2}(11), \\
\omega \mathrm{CH}_{2}(11)\end{array}$ \\
\hline$\nu \mathrm{CN}$ & $A$ & 914 & 8.3 & 2.8 & 1034 & $\mathrm{w}$ & 1041 & $\mathrm{w}$ & $\nu \mathrm{CN}(69), \nu \mathrm{CC}(19)$ \\
\hline$\rho \mathrm{CH}_{2}$ & $A$ & 865 & 31.6 & 1.0 & 911 & $\mathrm{~m}$ & 928 & $\mathrm{vw}$ & $\rho \mathrm{CH}_{2}(28), \rho^{\prime \prime} \mathrm{NH}_{3}(47), \omega \mathrm{COO}(28)$ \\
\hline$\nu \mathrm{CC}$ & $A$ & 831 & 111.9 & 6.5 & 893 & $\mathrm{~m}$ & 899 & $\mathrm{~s}$ & $\nu \mathrm{CC}(76), \nu \mathrm{CN}(19)$ \\
\hline$\delta \mathrm{COO}$ & $A$ & 648 & 3.0 & 3.4 & 698 & $\mathrm{~m}$ & 703 & $\mathrm{w}$ & $\delta \mathrm{COO}(61), \rho \mathrm{CH}_{2}(19), \nu \mathrm{CC}(11)$ \\
\hline$\omega \mathrm{COO}$ & $A$ & 571 & 5.0 & 3.8 & 608 & $\mathrm{w}$ & 608 & $\mathrm{w}$ & $\omega \mathrm{COO}(43), \rho \mathrm{CH}_{2}(24), \nu \mathrm{CC}(19), \delta \mathrm{COO}(16)$ \\
\hline$\delta \mathrm{CCO}$ & $A$ & 437 & 18.5 & 3.2 & 504 & $\mathrm{~m}$ & 499 & w & $\delta \mathrm{CCO}(44), \nu \mathrm{CC}(14)$ \\
\hline$\delta \mathrm{NCC}$ & $A$ & 320 & 32.7 & 0.2 & $358^{\mathrm{c}}$ & & 364 & $\mathrm{w}$ & $\delta \mathrm{NCC}(66), \delta \mathrm{CCO}(45)$ \\
\hline$\tau \mathrm{CN}$ & $A$ & 206 & 4.8 & 0.4 & $520^{\mathrm{d}}$ & sh & $509^{\mathrm{d}}$ & w & $\tau \mathrm{CN}$ \\
\hline$\tau \mathrm{CC}$ & $A$ & 119 & 2.1 & 0.3 & & & 186,171 & $\mathrm{w}, \mathrm{m}$ & $\tau \mathrm{CC}(46), \tau \mathrm{CN}(22), \omega \mathrm{COO}(13)$ \\
\hline
\end{tabular}

${ }^{a}$ Wavenumber values are in $\mathrm{cm}^{-1}$. IR int. (infrared intensities) $\mathrm{km} \cdot \mathrm{mol}^{-1}$ and $\mathrm{R}$ int. (Raman scattering activities) in $\AA^{4} /$ amu. $\nu$ stretching, $\delta$ bending, $\omega$ wagging, $\rho$ rocking, tw twisting, $\tau$ torsion, s symmetric, as asymmetric. The experimental intensities are only qualitative. PED: The number is parenthesis is the percent contribution of each internal mode for the total potential energy. Contributions $<10 \%$ are not shown. When only one internal mode in the PED have a contribution $>10 \%$, its value is ommited.

${ }^{\mathrm{b}}$ Higher component band of the complex structured band due to Fermi resonance interaction; other main relative maxima occur at $2830,2733,2622$ and $2532 \mathrm{~cm}{ }^{-1}$ (see text).

${ }^{\mathrm{c}}$ The predicted wavenumber for $\tau \mathrm{CN}$ is considerably lower than the experimental value, due to crystal packing forces (see text).

${ }^{\mathrm{d}}$ From Ref. [9]. 
Table 3

Spectra assignment and potential energy distribution for the neutral form of glycine ${ }^{\mathrm{a}}$

\begin{tabular}{|c|c|c|c|c|c|c|}
\hline \multirow[t]{2}{*}{ Assignment } & \multirow[t]{2}{*}{ Symmetry } & \multicolumn{2}{|l|}{ Calculated } & \multicolumn{3}{|c|}{ Experimental (MI-IR) } \\
\hline & & Wavenumber & IR int. & Wavenumber & Intensity & P.E.D. \\
\hline$\nu \mathrm{OH}$ & $A^{\prime}$ & 3607 & 110.1 & 3560 & $\mathrm{~m}$ & $\nu \mathrm{OH}$ \\
\hline$\nu_{\mathrm{as}} \mathrm{NH}_{2}$ & $A^{\prime \prime}$ & 3400 & 5.0 & 3410 & w & $\nu_{\mathrm{as}} \mathrm{NH}_{2}$ \\
\hline$\nu_{\mathrm{s}} \mathrm{NH}_{2}$ & $A^{\prime}$ & 3328 & 3.0 & 3200 & $\mathrm{w}$ & $\nu_{\mathrm{s}} \mathrm{NH}_{2}$ \\
\hline$\nu_{\mathrm{as}} \mathrm{CH}_{2}$ & $A^{\prime \prime}$ & 2915 & 15.5 & 2957 & w & $\nu_{\mathrm{as}} \mathrm{CH}_{2}$ \\
\hline$\nu_{\mathrm{s}} \mathrm{CH}_{2}$ & $A^{\prime}$ & 2881 & 19.9 & 2943 & w & $\nu_{\mathrm{s}} \mathrm{CH}_{2}$ \\
\hline$\nu \mathrm{C}=\mathrm{O}$ & $A^{\prime}$ & 1811 & 385.9 & 1780 & vs & $\nu \mathrm{C}=\mathrm{O}$ \\
\hline$\delta \mathrm{NH}_{2}$ & $A^{\prime}$ & 1648 & 25.4 & 1630,1623 & w & $\delta \mathrm{NH}_{2}(71), \omega \mathrm{NH}_{2}(24)$ \\
\hline$\delta \mathrm{CH}_{2}$ & $A^{\prime}$ & 1433 & 9.2 & 1428 & $\mathrm{w}$ & $\delta \mathrm{CH}_{2}$ \\
\hline$\omega \mathrm{CH}_{2}^{2}$ & $A^{\prime}$ & 1397 & 36.4 & 1385 & $\mathrm{w}$ & $\omega \mathrm{CH}_{2}(48), \nu \mathrm{CC}(16), \nu \mathrm{C}-\mathrm{O}(15)$ \\
\hline $\mathrm{twNH}_{2}$ & $A^{\prime \prime}$ & 1349 & 0.0 & 1373 & vw & $\mathrm{twNH}_{2}(47), \mathrm{twCH}_{2}(47)$ \\
\hline$\delta \mathrm{COH}$ & $A^{\prime}$ & 1288 & 19.4 & 1339 & w & $\delta \mathrm{COH}(38), \omega \mathrm{CH}_{2}(33), \nu \mathrm{C}-\mathrm{O}(10)$ \\
\hline$\nu \mathrm{C}-\mathrm{O}$ & $A^{\prime}$ & 1165 & 299.0 & 1100 & s & $\nu \mathrm{C}-\mathrm{O}(43), \delta \mathrm{COH}(34)$ \\
\hline $\mathrm{twCH}_{2}$ & $A^{\prime \prime}$ & 1154 & 2.1 & 1147 & $\mathrm{w}$ & $\mathrm{twCH}_{2}$ (49), $\mathrm{twNH}_{2}(32), \rho \mathrm{CH}_{2}$ (11) \\
\hline$\nu \mathrm{CN}$ & $A^{\prime}$ & 1096 & 39.9 & 1131 & $\mathrm{~m}$ & $\nu \mathrm{CN}$ \\
\hline$\omega \mathrm{NH}_{2}$ & $A^{\prime}$ & 930 & 198.2 & 882,880 & $\mathrm{~m}, \mathrm{w}$ & $\omega \mathrm{NH}_{2}(46), \nu \mathrm{CN}(17), \delta \mathrm{NH}_{2}(16)$ \\
\hline$\rho \mathrm{CH}_{2}{ }_{2}$ & $A^{\prime \prime}$ & 897 & 6.6 & 867,852 & $\mathrm{w}, \mathrm{w}$ & $\rho \mathrm{CH}_{2}(43), \mathrm{twNH}_{2}(26), \omega \mathrm{COO}(21)$ \\
\hline$\nu \mathrm{CC}$ & $A^{\prime \prime}$ & 809 & 80.3 & 799 & s & $\nu \mathrm{CC}(46), \omega \mathrm{NH}_{2}(13)$ \\
\hline$\tau \mathrm{C}-\mathrm{O}$ & $A^{\prime \prime}$ & 630 & 137.9 & 618 & $\mathrm{~m}$ & $\tau \mathrm{C}-\mathrm{O}(50), \omega \mathrm{COO}(30), \rho \mathrm{CH}_{2}(17)$ \\
\hline$\delta \mathrm{COO}$ & $A^{\prime}$ & 621 & 15.7 & 637 & $\mathrm{w}$ & $\delta \mathrm{COO}(50), \delta \mathrm{NCC}(16), \delta \mathrm{CC}=\mathrm{O}(12), \nu \mathrm{C}-\mathrm{O}(10)$ \\
\hline$\omega \mathrm{COO}$ & $A^{\prime \prime}$ & 491 & 38.6 & 498 & $\mathrm{w}$ & $\omega \mathrm{COO}(37), \tau \mathrm{C}-\mathrm{O}(40), \rho \mathrm{CH}_{2}(17)$ \\
\hline$\delta \mathrm{CC}=\mathrm{O}$ & $A^{\prime}$ & 448 & 34.1 & 463 & $\mathrm{~m}$ & $\delta \mathrm{CC}=\mathrm{O}(27), \delta \mathrm{COO}(34), \nu \mathrm{CC}(15), \delta \mathrm{NCC}(11)$ \\
\hline$\delta \mathrm{NCC}$ & $A^{\prime}$ & 250 & 10.8 & & & $\delta \mathrm{NCC}(47), \delta \mathrm{CC}=\mathrm{O}(47)$ \\
\hline$\tau \mathrm{CN}$ & $A^{\prime \prime}$ & 232 & 60.3 & & & $\tau \mathrm{CN}$ \\
\hline$\tau \mathrm{CC}$ & $A^{\prime \prime}$ & 74 & 5.2 & & & $\tau \mathrm{CC}(69), \tau \mathrm{CN}(13)$ \\
\hline
\end{tabular}

${ }^{\mathrm{a}}$ Wavenumber values are in $\mathrm{cm}^{-1}$. IR int. (infrared intensities) $\mathrm{km} \cdot \mathrm{mol}^{-1}$. $\nu$, stretching; $\delta$, bending; $\omega$, wagging; $\rho$, rocking; tw, twisting; $\tau$, torsion; s, symmetric; as, asymmetric. The experimental intensities are only qualitative. PED: The number is parenthesis is the percent contribution of each internal mode for the total potential energy. Contributions $<10 \%$ are not shown. When only one internal mode in the PED have a contribution $>10 \%$, its value is ommited.

though more elaborate studies on this problem are still required, our data are consonant with the following assignments:

(i) The infrared bands at 3176 and $3040 \mathrm{~cm}^{-1}$ (with the first also present in the infrared spectrum of crystalline $\mathrm{ND}_{3}$ deuterated zwitterionic glycine [5]) are mainly due to $\nu_{\mathrm{as}} \mathrm{NH}_{3}$ in Fermi resonance with the first overtone of the $\nu_{\mathrm{as}} \mathrm{COO}^{-}$mode (fundamental: $1596 \mathrm{~cm}^{-1}$ ). The doublet of bands at 3209 and $3165 \mathrm{~cm}^{-1}$ in the Raman spectrum of glycine acid salt has a similar origin (in the infrared spectrum of this compound only the lower frequency component can be observed, since the higher component band is buried within the more intense very broad $\nu \mathrm{OH}$ stretch band). This interpretation is reinforced by the observation in the infrared spectrum of crystalline $\mathrm{ND}_{3}$ deuterated zwitterionic glycine of a band at $3113 \mathrm{~cm}^{-1}$, due to the first overtone of the $\nu_{\text {as }} \mathrm{COO}^{-}$stretching, whose fundamental occurs at $1592 \mathrm{~cm}^{-1}$ [6].

(ii) In the Raman spectrum of crystalline glycine, the $\nu_{\text {as }} \mathrm{NH}_{3}$ modes give rise to a single broad band with maximum intensity at ca. $3149 \mathrm{~cm}^{-1}$ (close to the average frequency of the doublet of bands observed in the corresponding infrared spectrum).

(iii) The $\nu_{\mathrm{s}} \mathrm{NH}_{3}$ stretching is extensively involved in Fermi resonance with combination bands. For zwitterionic glycine, we tentatively interpret the maxima at 2899, 2830, 2732, 2622 and $2532 \mathrm{~cm}^{-1}$ to bands resulting mainly from Fermi interaction with $\delta_{\mathrm{s}} \mathrm{NH}_{3}+\nu_{\mathrm{s}} \mathrm{COO}^{-}\left(1505+1413 \mathrm{~cm}^{-1}\right)$, $\delta_{\mathrm{s}} \mathrm{NH}_{3}+\omega \mathrm{CH}_{2} \quad\left(1505+1334 \mathrm{~cm}^{-1}\right), \quad \delta_{\mathrm{as}} \mathrm{NH}_{3}+$ $\rho \mathrm{NH}_{3}\left(1615+1112 \mathrm{~cm}^{-1}\right), \delta_{\mathrm{as}} \mathrm{NH}_{3}+\nu \mathrm{CN}(1615$ $\left.+1034 \mathrm{~cm}^{-1}\right)$ and $\delta_{\text {as }} \mathrm{NH}_{3}+\rho \mathrm{CH}_{2}(1615+911$ 
Table 4

Spectra assignment and potential energy distribution for the glycine alkaline salt ${ }^{\mathrm{a}}$

\begin{tabular}{|c|c|c|c|c|c|c|c|c|c|}
\hline \multirow[t]{2}{*}{ Assignment } & \multirow[t]{2}{*}{ Symmetry } & \multicolumn{3}{|l|}{ Calculated } & \multicolumn{2}{|c|}{ Experimental (IR) } & \multicolumn{3}{|c|}{ Experimental (Raman) } \\
\hline & & Wavenumber & IR int. & $\mathrm{R}$ int. & Wavenumber & Intensity & Wavenumber & Intensity & P.E.D. \\
\hline$\nu_{\mathrm{as}} \mathrm{NH}_{2}$ & $A$ & 3342 & 4.1 & 48.4 & & & 3325 & $\mathrm{~m}$ & $\nu_{\mathrm{as}} \mathrm{NH}_{2}$ \\
\hline$\nu_{\mathrm{S}} \mathrm{NH}_{2}$ & $A$ & 3282 & 8.9 & 83.6 & & & 3212 & $\mathrm{~m}$ & $\nu_{\mathrm{s}} \mathrm{NH}_{2}$ \\
\hline$\nu_{\mathrm{as}} \mathrm{CH}_{2}$ & $A$ & 2872 & 82.5 & 80.3 & 2945 & $\mathrm{~m}$ & 2941 & vs & $\nu_{\mathrm{as}} \mathrm{CH}_{2}(80), \nu_{\mathrm{s}} \mathrm{CH}_{2}$ (19) \\
\hline$\nu_{\mathrm{s}} \mathrm{CH}_{2}$ & $A$ & 2831 & 87.2 & 108.7 & 2927 & $\mathrm{~m}$ & 2911 & vs & $\nu_{\mathrm{s}} \mathrm{CH}_{2}(80), \nu_{\mathrm{as}} \mathrm{CH}_{2}$ \\
\hline$\nu_{\mathrm{as}} \mathrm{COO}$ & $A$ & 1688 & 622.3 & 0.3 & 1597,1561 & s, vs & 1597,1572 & $\mathrm{w}, \mathrm{w}$ & $\nu_{\mathrm{as}} \mathrm{COO}$ \\
\hline$\delta \mathrm{NH}_{2}$ & $A$ & 1652 & 26.4 & 6.5 & 1630 & $\operatorname{sh}$ & 1630 & w & $\delta \mathrm{NH}_{2}$ \\
\hline$\delta \mathrm{CH}_{2}$ & $A$ & 1444 & 3.3 & 12.7 & 1431 & $\mathrm{~m}$ & 1439,1410 & $\mathrm{~s}, \mathrm{~s}$ & $\delta \mathrm{CH}_{2}$ \\
\hline$\nu_{\mathrm{s}} \mathrm{COO}$ & $A$ & 1392 & 234.3 & 4.2 & 1498,1457 & $\mathrm{~s}, \mathrm{~m}$ & 1462 & $\operatorname{sh}$ & $\begin{array}{l}\nu_{\mathrm{s}} \mathrm{COO}(56), \nu \mathrm{CC}(18) \\
\delta \mathrm{COO}(14)\end{array}$ \\
\hline $\mathrm{twNH}_{2}$ & $A$ & 1344 & 23.7 & 10.7 & 1355,1336 & $\mathrm{w}, \mathrm{w}$ & 1358,1314 & $\mathrm{~m}, \mathrm{~m}$ & $\mathrm{twNH}_{2}(53), \mathrm{twCH}_{2}$ (33) \\
\hline$\omega \mathrm{CH}_{2}$ & $A$ & 1292 & 115.1 & 2.9 & 1270 & $\mathrm{~m}$ & & & $\omega \mathrm{CH}_{2}(70), \nu_{\mathrm{s}} \mathrm{COO}(12)$ \\
\hline $\mathrm{twCH}_{2}$ & $A$ & 1118 & 11.5 & 3.4 & 1159 & $\mathrm{w}$ & 1168,1122 & $\mathrm{w}, \mathrm{m}$ & $\mathrm{twCH}_{2}(48), \mathrm{twNH}_{2}(23)$ \\
\hline$\omega \mathrm{NH}_{2}$ & $A$ & 1094 & 2.0 & 0.7 & 1069 & $\mathrm{w}$ & 1079 & w & $\omega \mathrm{NH}_{2}(47), \nu \mathrm{CN}(17)$ \\
\hline$\nu \mathrm{CN}$ & $A$ & 997 & 4.6 & 0.5 & 1009 & $\mathrm{w}$ & 1004 & $\mathrm{w}$ & $\nu \mathrm{CN}(54), \omega \mathrm{NH}_{2}(28)$ \\
\hline$\rho \mathrm{CH}_{2}$ & $A$ & 912 & 0.4 & 0.4 & 944,900 & $\mathrm{w}, \mathrm{w}$ & 909 & vs & $\begin{array}{l}\rho \mathrm{CH}_{2}(39), \mathrm{twNH}_{2}(25), \\
\omega \mathrm{COO}(25)\end{array}$ \\
\hline$\nu \mathrm{CC}$ & $A$ & 855 & 9.6 & 0.1 & 865,827 & $\mathrm{w}$ & & & $\begin{array}{l}\nu \mathrm{CC}(37), \delta \mathrm{COO}(33) \\
\nu_{\mathrm{s}} \mathrm{COO}(12)\end{array}$ \\
\hline$\delta \mathrm{COO}$ & $A$ & 661 & 3.9 & 0.7 & 674 & w & 636 & $\mathrm{w}$ & $\begin{array}{c}\delta \mathrm{COO}(36), \delta \mathrm{NCC}(21) \\
\nu \mathrm{CC}(12), \delta \mathrm{CCO}(10)\end{array}$ \\
\hline$\omega \mathrm{COO}$ & $A$ & 561 & 13.5 & 2.6 & 622 & $\mathrm{~m}$ & 582 & w & $\omega \mathrm{COO}(49), \rho \mathrm{CH}_{2}(30)$ \\
\hline$\delta \mathrm{CCO}$ & $A$ & 474 & 10.9 & 4.5 & 519 & w & 517 & $\mathrm{~s}$ & $\begin{array}{l}\delta \mathrm{CCO}(41), \nu \mathrm{CC}(18) \\
\delta \mathrm{NCC}(11)\end{array}$ \\
\hline$\delta \mathrm{NCC}$ & $A$ & 280 & 16.1 & 0.3 & & & 261 & w & $\delta \mathrm{NCC}(54), \delta \mathrm{CCO}(38)$ \\
\hline$\tau \mathrm{CN}$ & $A$ & 180 & 67.9 & 2.9 & & & 239,206 & $\mathrm{~m}, \mathrm{sh}$ & $\tau \mathrm{CN}$ \\
\hline$\tau \mathrm{CC}$ & $A$ & 73 & 3.9 & 0.2 & & & & & $\tau \mathrm{CC}(63), \tau \mathrm{CN}(12)$ \\
\hline
\end{tabular}

${ }^{a}$ Wavenumber values are in $\mathrm{cm}^{-1}$. IR int. (infrared intensities) $\mathrm{km} \cdot \mathrm{mol}^{-1}$ and $\mathrm{R}$ int. (Raman scattering activities) in $\AA^{4} /$ amu. $\nu$, stretching; $\delta$, bending; $\omega$, wagging; $\rho$, rocking; tw, twisting; $\tau$, torsion; s, symmetric; as, asymmetric. The experimental intensities are only qualitative. PED: The number in parentheses is the percent contribution of each internal mode for the total potential energy. Contributions $<10 \%$ are not shown. When only one internal mode in the PED has a contribution $>10 \%$, its value is ommited. 
Table 5

Calculated spectra and potential energy distribution for the glycine acid salt (monomer 'model') ${ }^{\mathrm{a}}$

\begin{tabular}{|c|c|c|c|c|c|}
\hline Assignment & Symmetry & Wavenumber & IR int. & $\mathrm{R}$ int. & P.E.D. \\
\hline$\nu \mathrm{OH}$ & $A^{\prime}$ & 3558 & 244.9 & 69.4 & $\nu \mathrm{OH}$ \\
\hline$\nu_{\mathrm{as}} \mathrm{NH}_{3}$ & $A^{\prime}$ & 3320 & 155.3 & 37.9 & $\nu_{\mathrm{as}} \mathrm{NH}_{3}$ \\
\hline$\nu_{\mathrm{as}} \mathrm{NH}_{3}$ & $A^{\prime \prime}$ & 3303 & 167.5 & 23.7 & $\nu_{\mathrm{as}} \mathrm{NH}_{3}$ \\
\hline$\nu_{\mathrm{s}} \mathrm{NH}_{3}$ & $A^{\prime}$ & 3223 & 77.2 & 54.8 & $\nu_{\mathrm{s}} \mathrm{NH}_{3}$ \\
\hline$\nu_{\mathrm{as}} \mathrm{CH}_{2}$ & $A^{\prime \prime}$ & 2988 & 2.9 & 47.8 & $\nu_{\mathrm{as}} \mathrm{CH}_{2}$ \\
\hline$\nu_{\mathrm{s}} \mathrm{CH}_{2}$ & $A^{\prime}$ & 2929 & 3.9 & 79.0 & $\nu_{\mathrm{s}} \mathrm{CH}_{2}$ \\
\hline$\delta_{\mathrm{as}} \mathrm{NH}_{3}$ & $A^{\prime \prime}$ & 1608 & 65.0 & 6.1 & $\delta_{\mathrm{as}} \mathrm{NH}_{3}$ \\
\hline$\delta_{\mathrm{s}} \mathrm{NH}_{3}$ & $A^{\prime}$ & 1521 & 187.8 & 0.5 & $\delta_{\mathrm{s}} \mathrm{NH}_{3}$ \\
\hline$\nu \mathrm{C}-\mathrm{O}$ & $A^{\prime}$ & 1440 & 106.7 & 4.3 & $\nu \mathrm{C}-\mathrm{O}(15), \delta \mathrm{CH}_{2}(38), \nu \mathrm{CC}(18), \omega \mathrm{CH}_{2}(16)$ \\
\hline$\delta \mathrm{CH}_{2}$ & $A^{\prime}$ & 1435 & 41.3 & 4.3 & $\delta \mathrm{CH}_{2}(53), \omega \mathrm{CH}_{2}(21)$ \\
\hline$\omega \mathrm{CH}_{2}$ & $A^{\prime}$ & 1311 & 42.3 & 1.5 & $\omega \mathrm{CH}_{2}(46), \delta \mathrm{COH}(18), \nu \mathrm{C}-\mathrm{O}(17)$ \\
\hline $\mathrm{twCH}_{2}$ & $A^{\prime \prime}$ & 1286 & 17.3 & 5.6 & $\mathrm{twCH}_{2}(67), \rho \mathrm{NH}_{3}(21)$ \\
\hline$\nu \mathrm{CN}$ & $A^{\prime}$ & 939 & 8.2 & 3.5 & $\nu \mathrm{CN}(74), \nu \mathrm{CC}(13)$ \\
\hline$\rho \mathrm{CH}_{2}$ & $A^{\prime \prime}$ & 873 & 40.5 & 0.4 & $\rho \mathrm{CH}_{2}(33), \rho \mathrm{NH}_{3}(42), \omega \mathrm{COO}(15)$ \\
\hline$\nu \mathrm{CC}$ & $A^{\prime}$ & 807 & 45.4 & 9.9 & $\nu \mathrm{CC}(44), \nu \mathrm{CN}(11), \rho \mathrm{NH}_{3}(11)$ \\
\hline$\tau \mathrm{C}-\mathrm{O}$ & $A^{\prime \prime}$ & 632 & 143.6 & 0.3 & $\tau \mathrm{C}-\mathrm{O}(67), \omega \mathrm{COO}(22)$ \\
\hline$\delta \mathrm{COO}$ & $A^{\prime}$ & 608 & 25.7 & 2.9 & $\delta \mathrm{COO}(50), \delta \mathrm{NCC}(16), \delta \mathrm{CC}=\mathrm{O}(12)$ \\
\hline$\omega \mathrm{COO}$ & $A^{\prime \prime}$ & 509 & 44.9 & 2.7 & $\omega \mathrm{COO}(58), \rho \mathrm{CH}_{2}(23)$ \\
\hline$\delta \mathrm{CC}=\mathrm{O}$ & $A^{\prime}$ & 448 & 57.1 & 1.5 & $\delta \mathrm{CC}=\mathrm{O}(29), \delta \mathrm{COO}(29), \delta \mathrm{NCC}(14)$ \\
\hline$\delta \mathrm{NCC}$ & $A^{\prime}$ & 265 & 25.7 & 0.1 & $\delta \mathrm{NCC}(48), \delta \mathrm{CC}=\mathrm{O}(47)$ \\
\hline$\tau \mathrm{CN}$ & $A^{\prime \prime}$ & 161 & 0.9 & 0.0 & $\tau \mathrm{CN}(65), \tau \mathrm{CC}(22)$ \\
\hline$\tau \mathrm{CC}$ & $A^{\prime \prime}$ & 48 & 5.3 & 0.1 & $\tau \mathrm{CC}(44), \tau \mathrm{CN}(38)$ \\
\hline
\end{tabular}

${ }^{\mathrm{a}}$ Wavenumber values are in $\mathrm{cm}^{-1}$. IR int. (infrared intensities) $\mathrm{km} \cdot \mathrm{mol}^{-1}$ and $\mathrm{R}$ int. (Raman scattering activities) in $\AA^{4} / \mathrm{amu}$. $\nu$, stretching; $\delta$, bending; $\omega$, wagging; $\rho$, rocking; tw, twisting; $\tau$, torsion; s, symmetric; as, asymmetric. P.E.D.: The number in parentheses is the percent contribution of each internal mode for the total potential energy. Contributions $<10 \%$ are not shown. When only one internal mode in the PED has a contribution $>10 \%$, its value is ommited.

$\left.\mathrm{cm}^{-1}\right)$. In the acid salt, the corresponding bands appear at 3003, 2840, 2678, 2595 and $2525 \mathrm{~cm}^{-1}$, with the dominant band at $3003 \mathrm{~cm}^{-1}$.

(iv) The frequencies of the $\nu \mathrm{NH}_{2}$ stretching modes in the alkaline salt of glycine and, in particular, the $\nu \mathrm{NH}_{3}$ vibrations in both crystalline glycine and its acid salt are considerably overestimated by the calculations, since these groups participate in intermolecular hydrogen bonding that is not properly considered in the calculations. However, the calculations are able to predict correctly the general trends, both with respect to relative positions of the various $\nu \mathrm{NH}$ vibrations within the same molecule and the relative values for the same mode in different molecules (see Tables 2-6).

In the infrared spectrum of the crystalline glycine, in addition to the bands due to the $\nu \mathrm{NH}_{3}$ and $\nu \mathrm{CH}_{2}$ stretching modes, a band of medium intensity is also observed at $2117 \mathrm{~cm}^{-1}$, and is here assigned to the combination mode $\delta_{\text {as }} \mathrm{NH}_{3}+\tau \mathrm{NH}_{3}$. As discussed in Section 3.2 the fundamentals are assigned to the bands at 1615 and $520 \mathrm{~cm}^{-1}$.

\subsection{Region $2000-1400 \mathrm{~cm}^{-1}$}

This region of the spectrum includes the carbonyl stretching band $(\nu \mathrm{C}=\mathrm{O}), \mathrm{NH}_{2}$ and $\mathrm{NH}_{3}$ bending modes, $\mathrm{CH}_{2}$ scissoring and the two carboxylate stretching modes $\left(\nu_{\mathrm{as}} \mathrm{COO}^{-}\right.$and $\left.\nu_{\mathrm{s}} \mathrm{COO}^{-}\right)$.

The carbonyl stretching band is present in both neutral glycine and acid salt spectra and can be assigned to the intense infrared bands at $1780 \mathrm{~cm}^{-1}$ (glycine) and $1745 \mathrm{~cm}^{-1}$ (acid salt; $B_{\mathrm{u}}$ mode) and to the doublet of bands at 1737 and $1717 \mathrm{~cm}^{-1}$ in the 
Table 6

Spectra assignment and potential energy distribution for the glycine acid salt dimer ${ }^{\mathrm{a}}$

\begin{tabular}{|c|c|c|c|c|c|c|c|c|c|}
\hline \multirow[t]{2}{*}{ Assignment } & \multirow[t]{2}{*}{ Symmmetry } & \multicolumn{3}{|l|}{ Calculated } & \multicolumn{2}{|c|}{ Experimental (IR) } & \multicolumn{3}{|c|}{ Experimental (Raman) } \\
\hline & & Wavenumber & IR int. & $\mathrm{R}$ int. & Wavenumber & Intensity & Wavenumber & Intensity & P.E.D. \\
\hline$\nu \mathrm{OH}$ & $B_{u}$ & 3437 & 1590.0 & & 3439 & vs & & & $\nu \mathrm{OH}$ \\
\hline$\nu \mathrm{OH}$ & $A_{g}$ & 3404 & & 238.8 & & & & & $\nu \mathrm{OH}$ \\
\hline$\nu_{\mathrm{as}} \mathrm{NH}_{3}$ & $A_{g}$ & 3303 & & 78.2 & & & 3209,3165 & $\mathrm{~m}, \mathrm{~m}$ & $\nu_{\mathrm{as}} \mathrm{NH}_{3}$ \\
\hline$\nu_{\mathrm{as}} \mathrm{NH}_{3}$ & $B_{u}$ & 3302 & 353.5 & & 3196 & s & & & $\nu_{\mathrm{as}} \mathrm{NH}_{3}$ \\
\hline$\nu_{\mathrm{as}} \mathrm{NH}_{3}$ & $A_{u}$ & 3302 & 333.4 & & 3196 & s & & & $\nu_{\mathrm{as}} \mathrm{NH}_{3}$ \\
\hline$\nu_{\mathrm{as}} \mathrm{NH}_{3}$ & $B_{g}$ & 3302 & & 45.7 & & & 3209,3165 & $\mathrm{~m}, \mathrm{~m}$ & $\nu_{\mathrm{as}} \mathrm{NH}_{3}$ \\
\hline$\nu_{\mathrm{S}} \mathrm{NH}_{3}$ & $A_{g}$ & 3216 & & 116.4 & & & 3020 & $\mathrm{~m}$ & $\nu_{\mathrm{s}} \mathrm{NH}_{3}$ \\
\hline$\nu_{\mathrm{s}} \mathrm{NH}_{3}$ & $B_{u}^{s}$ & 3216 & 189.1 & & 3003 & vs & & & $\nu_{\mathrm{s}} \mathrm{NH}_{3}$ \\
\hline$\nu_{\mathrm{as}} \mathrm{CH}_{2}$ & $A_{u}$ & 2982 & 13.2 & & & & & & $\nu_{\mathrm{as}} \mathrm{CH}_{2}$ \\
\hline$\nu_{\mathrm{as}} \mathrm{CH}_{2}$ & $B_{g}$ & 2982 & & 97.2 & & & 3001 & vs & $\nu_{\mathrm{as}} \mathrm{CH}_{2}$ \\
\hline$\nu_{\mathrm{s}} \mathrm{CH}_{2}$ & $A_{g}$ & 2925 & & 159.1 & & & 2968 & vs & $\nu_{\mathrm{s}} \mathrm{CH}_{2}$ \\
\hline$\nu_{\mathrm{s}} \mathrm{CH}_{2}$ & $B_{u}^{\circ}$ & 2925 & 19.0 & & & & & & $\nu_{\mathrm{s}} \mathrm{CH}_{2}$ \\
\hline$\nu \mathrm{C}=\mathrm{O}$ & $B_{u}$ & 1787 & 919.2 & & 1745 & vs & & & $\nu \mathrm{C}=\mathrm{O}(89), \nu \mathrm{C}-\mathrm{O}(14)$ \\
\hline$\nu \mathrm{C}=\mathrm{O}$ & $A_{g}$ & 1756 & & 6.7 & & & 1737,1717 & $\mathrm{~m}, \mathrm{w}$ & $\nu \mathrm{C}=\mathrm{O}(90), \nu \mathrm{C}-\mathrm{O}(11)$ \\
\hline$\delta_{\text {as }} \mathrm{NH}_{3}$ & $A_{g}^{b}$ & 1614 & & 9.6 & & & 1601 & w & $\delta_{\mathrm{as}} \mathrm{NH}_{3}$ \\
\hline$\delta_{\mathrm{as}} \mathrm{NH}_{3}$ & $B_{u}^{\delta}$ & 1614 & 84.5 & & 1625 & $\mathrm{~m}$ & & & $\delta_{\mathrm{as}} \mathrm{NH}_{3}$ \\
\hline$\delta_{\text {as }} \mathrm{NH}_{3}$ & $A_{u}$ & 1612 & 137.6 & & 1594 & $\mathrm{~m}$ & & & $\delta_{\mathrm{as}} \mathrm{NH}_{3}$ \\
\hline$\delta_{\text {as }} \mathrm{NH}_{3}$ & $B_{g}$ & 1612 & & 13.2 & & & 1585 & $\mathrm{~m}$ & $\delta_{\mathrm{as}} \mathrm{NH}_{3}$ \\
\hline$\delta_{\mathrm{s}} \mathrm{NH}_{3}$ & $A_{g}$ & 1526 & & 0.8 & & & 1494 & $\mathrm{~m}$ & $\delta_{\mathrm{s}} \mathrm{NH}_{3}$ \\
\hline$\delta_{\mathrm{s}} \mathrm{NH}_{3}$ & $B_{u}^{\circ}$ & 1525 & 363.4 & & 1486 & s & & & $\delta_{\mathrm{s}} \mathrm{NH}_{3}$ \\
\hline$\nu \mathrm{C}-\mathrm{O}$ & $A_{g}$ & 1464 & & 5.8 & & & 1445 & w & $\begin{array}{c}\nu \mathrm{C}-\mathrm{O}(32), \omega \mathrm{CH}_{2}(26) \\
\nu \mathrm{CC}(21), \delta \mathrm{COO}(12)\end{array}$ \\
\hline$\nu \mathrm{C}-\mathrm{O}$ & $B_{u}$ & 1461 & 301.0 & & 1437 & s & & & $\begin{array}{c}\nu \mathrm{C}-\mathrm{O}(29), \omega \mathrm{CH}_{2}(30) \\
\nu \mathrm{CC}(24), \delta \mathrm{COO}(12)\end{array}$ \\
\hline$\delta \mathrm{CH}_{2}$ & $A_{g}$ & 1431 & & 16.2 & & & 1421 & $\mathrm{~m}$ & $\delta \mathrm{CH}_{2}$ \\
\hline$\delta \mathrm{CH}_{2}$ & $B_{u}^{s}$ & 1431 & 60.6 & & 1421 & $\mathrm{~s}$ & & & $\delta \mathrm{CH}_{2}$ \\
\hline$\omega \mathrm{CH}_{2}$ & $A_{g}$ & 1344 & & 7.6 & & & 1342 & w & $\omega \mathrm{CH}_{2}(53), \delta \mathrm{COH}(23)$ \\
\hline$\omega \mathrm{CH}_{2}$ & $B_{u}^{8}$ & 1328 & 96.4 & & 1332 & $\mathrm{~m}$ & & & $\begin{array}{l}\omega \mathrm{CH}_{2}(52), \delta \mathrm{COH}(21), \\
\nu \mathrm{C}-\mathrm{O}(14)\end{array}$ \\
\hline $\mathrm{twCH}_{2}$ & $A_{u}$ & 1292 & 31.1 & & 1313 & w & & & $\mathrm{twCH}_{2}(60), \rho \mathrm{NH}_{3}(17)$ \\
\hline $\mathrm{twCH}_{2}$ & $B_{g}$ & 1292 & & 11.2 & & & 1315 & $\mathrm{~m}$ & $\mathrm{twCH}_{2}(60), \rho \mathrm{NH}_{3}$ (17) \\
\hline$\delta \mathrm{COH}$ & $A_{g}$ & 1234 & & 7.3 & & & 1219 & $\mathrm{~m}$ & $\delta \mathrm{COH}(46), \nu \mathrm{C}-\mathrm{O}(40)$ \\
\hline$\delta \mathrm{COH}$ & $B_{u}^{s}$ & 1226 & 380.9 & & 1254,1216 & $\mathrm{~s}, \mathrm{~s}$ & & & $\delta \mathrm{COH}(64), \nu \mathrm{C}-\mathrm{O}(31)$ \\
\hline$\rho \mathrm{NH}_{3}$ & $A_{u}$ & 1085 & 0.6 & & 1115 & w & & & $\begin{array}{c}\rho \mathrm{NH}_{3}(34), \mathrm{twCH}_{2}(32), \\
\rho \mathrm{CH} 2(26), \omega \mathrm{COO}(14)\end{array}$ \\
\hline$\rho \mathrm{NH}_{3}$ & $B_{g}$ & 1085 & & 1.3 & & & 1125 & $\mathrm{w}$ & $\begin{array}{c}\rho \mathrm{NH}_{3}(34), \mathrm{twCH}_{2}(32), \\
\rho \mathrm{CH} 2(26), \omega \mathrm{COO}(14)\end{array}$ \\
\hline
\end{tabular}




\begin{tabular}{|c|c|c|c|c|c|c|c|c|c|}
\hline$\rho \mathrm{NH}_{3}$ & $A_{g}$ & 1069 & & 3.4 & & & 1108 & $\mathrm{~m}$ & $\rho \mathrm{NH}_{3}(65), \omega \mathrm{CH}_{2}(15)$ \\
\hline$\rho \mathrm{NH}_{3}$ & $B_{u}^{g}$ & 1069 & 98.3 & & 1103 & $\mathrm{~m}$ & & & $\rho \mathrm{NH}_{3}(65), \omega \mathrm{CH}_{2}(14)$ \\
\hline$\nu \mathrm{CN}$ & $A_{g}$ & 952 & & 7.1 & & & 1047 & $\mathrm{~m}$ & $\nu \mathrm{CN}(72), \nu \mathrm{CC}(15)$ \\
\hline$\nu \mathrm{CN}$ & $B_{u}^{g}$ & 951 & 16.3 & & 1040,1027 & $\mathrm{w}, \mathrm{w}$ & & & $\nu \mathrm{CN}(72), \nu \mathrm{CC}(14)$ \\
\hline$\rho \mathrm{CH}_{2}$ & $A_{u}$ & 875 & 111.9 & & 912,900 & $\mathrm{sh}, \mathrm{m}$ & & & $\rho \mathrm{CH}_{2}(35), \rho \mathrm{NH}_{3}(45), \omega \mathrm{COO}(20)$ \\
\hline$\rho \mathrm{CH}_{2}$ & $B_{g}$ & 873 & & 0.8 & & & 904 & $\mathrm{~m}$ & $\rho \mathrm{CH}_{2}(36), \rho \mathrm{NH}_{3}(46), \omega \mathrm{COO}(20)$ \\
\hline$\nu \mathrm{CC}$ & $B_{u}^{\delta}$ & 823 & 73.8 & & 870,857 & $\mathrm{w}, \mathrm{w}$ & & & $\nu \mathrm{CC}(42), \nu \mathrm{CN}(15), \delta \mathrm{COO}(14), \rho \mathrm{NH}_{3}(12)$ \\
\hline$\nu \mathrm{CC}$ & $A_{g}$ & 819 & & 17.8 & & & 860 & vs & $\nu \mathrm{CC}(44), \nu \mathrm{CN}(15), \delta \mathrm{COO}(13), \rho \mathrm{NH}_{3}(12)$ \\
\hline$\tau \mathrm{C}-\mathrm{O}$ & $A_{u}^{\circ}$ & 780 & 295.3 & & 804 & w & & & $\tau \mathrm{C}-\mathrm{O}(73), \delta_{\mathrm{op}}($ ring $)(12)$ \\
\hline$\tau \mathrm{C}-\mathrm{O}$ & $B_{g}$ & 737 & & 0.6 & & & & & $\tau \mathrm{C}-\mathrm{O}(90)$ \\
\hline$\delta \mathrm{COO}$ & $B_{u}^{g}$ & 622 & 50.6 & & 675,640 & $\mathrm{w}, \mathrm{w}$ & & & $\delta \mathrm{COO}(48), \delta \mathrm{NCC}(15), \delta \mathrm{CC}=\mathrm{O}(11)$ \\
\hline$\delta \mathrm{COO}$ & $A_{g}$ & 618 & & 5.6 & & & 637 & $\mathrm{~m}$ & $\delta \mathrm{COO}(51), \delta \mathrm{NCC}(14), \delta \mathrm{CC}=\mathrm{O}(10)$ \\
\hline$\omega \mathrm{COO}$ & $B_{g}$ & 555 & & 2.3 & & & 571 & $\mathrm{w}$ & $\omega \mathrm{COO}(63), \rho \mathrm{CH}_{2}(23)$ \\
\hline$\omega \mathrm{COO}$ & $A_{u}$ & 545 & 3.9 & & $\approx 540$ & $\mathrm{vw}$ & & & $\omega \mathrm{COO}(61), \rho \mathrm{CH}_{2}$ \\
\hline$\delta \mathrm{CC}=\mathrm{O}$ & $B_{u}$ & 464 & 65.4 & & 496 & $\mathrm{w}$ & & & $\delta \mathrm{CC}=\mathrm{O}(30), \delta \mathrm{COO}(21), \nu \mathrm{CC}(11)$ \\
\hline$\delta \mathrm{CC}=\mathrm{O}$ & $A_{g}$ & 457 & & 5.1 & & & 498 & $\mathrm{~m}$ & $\delta \mathrm{CC}=\mathrm{O}(31), \delta \mathrm{COO}(20), \delta \mathrm{NCC}(13)$ \\
\hline$\delta \mathrm{NCC}$ & $B_{u}$ & 269 & 119.3 & & & & & & $\delta \operatorname{NCC}(58), \delta \mathrm{CCO}(35), \nu \mathrm{H} \ldots \mathrm{O}(19)$ \\
\hline$\delta \mathrm{NCC}$ & $A_{g}$ & 269 & & 0.1 & & & 296 & $\mathrm{~m}$ & $\delta \mathrm{NCC}(53), \delta \mathrm{CCO}(42), \delta \mathrm{C}=\mathrm{O} \ldots \mathrm{H}(17)$ \\
\hline$\tau \mathrm{CN}$ & $B_{g}^{8}$ & 167 & & 0.1 & & & 178 & $\mathrm{~m}$ & $\tau \mathrm{CN}(56), \tau \mathrm{CC}(13)$ \\
\hline$\tau \mathrm{CN}$ & $A_{u}$ & 166 & 0.1 & & & & & & $\tau \mathrm{CN}(54), \tau \mathrm{CC}(15)$ \\
\hline$\delta \mathrm{C}=\mathrm{O} \ldots \mathrm{H}$ & $A_{g}$ & 114 & & 0.3 & & & 135 & $\mathrm{~m}$ & $\delta \mathrm{C}=\mathrm{O} \ldots \mathrm{H}(48), \nu \mathrm{H} \ldots \mathrm{O}(33)$ \\
\hline$\tau \mathrm{C}=\mathrm{O}$ & $B_{g}^{8}$ & 98 & & 0.4 & & & & & $\tau \mathrm{C}=\mathrm{O}(76), \tau \mathrm{C}-\mathrm{O}(19)$ \\
\hline$\nu \mathrm{H} \ldots \mathrm{O}$ & $A_{g}^{8}$ & 95 & & 0.0 & & & & & $\nu \mathrm{H} \ldots \mathrm{O}(55), \delta \mathrm{C}=\mathrm{O} \ldots \mathrm{H}(26)$ \\
\hline$\tau \mathrm{C}=\mathrm{O}$ & $A_{u}$ & 80 & 6.4 & & & & & & $\tau \mathrm{C}=\mathrm{O}(18), \tau \mathrm{CC}(31), \tau \mathrm{CN}(26), \tau \mathrm{C}-\mathrm{O}$ \\
\hline$\nu \mathrm{H} \ldots \mathrm{O}$ & $B_{u}$ & 78 & 20.0 & & & & & & $\nu \mathrm{H} \ldots \mathrm{O}(81), \delta \mathrm{CCO}(15)$ \\
\hline$\tau \mathrm{CC}$ & $B_{g}$ & 61 & & 0.1 & & & & & $\tau \mathrm{CC}(50), \tau \mathrm{CN}(32)$ \\
\hline$\tau \mathrm{CC}$ & $A_{u}$ & 51 & 4.9 & & & & & & $\tau \mathrm{CC}(33), \tau \mathrm{C}=\mathrm{O}(26), \tau \mathrm{CN}(19), \delta_{\mathrm{op}}($ ring) $(11)$ \\
\hline$\delta_{\text {op }}($ ring $)$ & $A_{u}$ & 39 & 37.5 & & & & & & $\delta_{\text {op }}($ ring $)(41), \tau \mathrm{CC}(32), \tau \mathrm{CN}(11), \tau \mathrm{C}-\mathrm{O}(10)$ \\
\hline
\end{tabular}

${ }^{\mathrm{a}}$ Wavenumber values are in $\mathrm{cm}^{-1}$. IR int. (infrared intensities) $\mathrm{km} \cdot \mathrm{mol}^{-1}$ and $\mathrm{R}$ int. (Raman scattering activities) in $\AA^{4} /$ amu. $\nu$, stretching; $\delta$, bending; $\omega$, wagging; $\rho$, rocking; tw, twisting; $\tau$, torsion; s, symmetric; as, asymmetric. The experimental intensities are only qualitative. PED: The number in parentheses is the percent contribution of each internal mode for the total potential energy. Contributions $<10 \%$ are not shown. When only one internal mode in the PED have a contribution $>10 \%$, its value is ommited. 
Raman spectrum of the later compound ( $A_{\mathrm{g}}$ mode). The appearance of the doublet of bands in the Raman spectrum is probably due to a Fermi resonance involving the carbonyl stretching fundamental and the first overtone of the $\mathrm{C}-\mathrm{C}$ stretching mode $\left(A_{\mathrm{g}}\right.$ symmetry) whose fundamental vibration leads to the intense Raman band at $860 \mathrm{~cm}^{-1}$. As is commonly observed [27-31], the calculations considerably overestimate the frequency of the $\nu \mathrm{C}=\mathrm{O}$ mode, since they do not fully account for intermolecular effects that tend to reduce the frequency of this vibration (for glycine, polarization effects due to matrix/solute interactions [27-29] were not considered, while interdimer interactions [30,31] were neglected in the acid salt). In the case of the glycine acid salt, however, comparison of predicted frequencies for $\nu \mathrm{C}=\mathrm{O}$ calculated using monomer and dimer 'models' (see Tables 5 and 6) clearly shows that the calculated frequency for this mode is considerably improved in the later, where intermolecular association is partially considered.

As with the $\nu \mathrm{C}=\mathrm{O}$ mode, the calculations also overestimate the frequency of the antisymmetrical $\mathrm{COO}^{-}$stretching mode of the zwitterionic form and of the alkaline salt, while they underestimate the frequencies of the symmetrical $\mathrm{COO}^{-}$stretching vibration (see Tables 2 and 4). This results could be anticipated, since the calculations (for isolated molecules) do not take properly into account intermolecular interactions acting in the crystalline state that tend to make the two $\mathrm{C}-\mathrm{O}$ bonds more similar and, thus, lead to an increase of the $\nu_{\mathrm{s}} \mathrm{COO}^{-}$and decrease of $\nu_{\mathrm{as}} \mathrm{COO}^{-}$mode frequencies [30-32]. The assignment of the $\mathrm{COO}^{-}$stretching modes is made easier because these vibrations are predicted by the calculations to give rise to very intense infrared bands, whose counterparts in the Raman spectra are relatively weak.

The $\mathrm{NH}_{2}$ and $\mathrm{NH}_{3}$ bending modes were assigned easily, despite, in general, that some of the bands originated in these vibrations cannot be observed in the infrared spectra due to extensive overlap with the very intense $\nu_{\mathrm{as}} \mathrm{COO}$ band (they are, however, clearly observed in Raman).

The assignment of the $\mathrm{CH}_{2}$ scissoring band does not present any major difficulty in the spectra of glycine and is ascribed to the infrared band at 1428 $\mathrm{cm}^{-1}$ for the neutral form and to the doublet at 1460 and $1444 \mathrm{~cm}^{-1}$ (1462 and $1446 \mathrm{~cm}^{-1}$ in the Raman spectrum) for the zwitterion. In the alkaline salt, this mode gives rise to the infrared band at $1431 \mathrm{~cm}^{-1}$ and to the Raman doublet at 1439 and $1410 \mathrm{~cm}^{-1}$, while in the acid salt it gives the band at $1421 \mathrm{~cm}^{-1}$ (both in the infrared and Raman spectra).

Because carboxylic acid group intra-dimer interactions in the acid salt of glycine considerably increase the double bond character of the $\mathrm{C}-\mathrm{O}(\mathrm{H})$ bond when compared with a pure carboxylic acid moiety, the $\nu \mathrm{C}-\mathrm{O}$ vibration in this molecule occurs at a much higher frequency than in the isolated neutral form of glycine. We assign the infrared band at $1437 \mathrm{~cm}^{-1}$ (Raman: $1445 \mathrm{~cm}^{-1}$ ) of the acid salt to this mode (infrared, $B_{\mathrm{u}}$ mode; Raman, $A_{\mathrm{g}}$ mode), while in the MI-IR spectrum of the neutral form of glycine this mode gives rise to the band at 1100 $\mathrm{cm}^{-1}$. The corresponding calculated values for this mode are: glycine acid salt: $1464 \mathrm{~cm}^{-1}\left(A_{\mathrm{g}}\right.$ mode), $1461 \mathrm{~cm}^{-1}$ ( $B_{\mathrm{u}}$ mode); neutral glycine: $\left.1165 \mathrm{~cm}^{-1}\right)$. Note that as with the remaining $\mathrm{C}-\mathrm{O}$ stretching modes, the predicted frequencies are overestimated by the calculations.

\subsection{Region $1400-800 \mathrm{~cm}^{-1}$}

Most of the bands in this region are due to the wagging, twisting and rocking modes of the $\mathrm{CH}_{2}$, $\mathrm{NH}_{2}$ and $\mathrm{NH}_{3}$ groups. When the $\mathrm{CH}_{2}$ group is placed in an adjacent position to the $\mathrm{NH}_{2}$ or $\mathrm{NH}_{3}$ fragments, the vibrations become strongly coupled. This is reflected in the extensive mixing of these coordinates in the potential energy distribution calculated for most of these modes (see Tables 2-6).

For molecules containing the carboxylic acid group, the band due to $\delta \mathrm{COH}$ can also be found in this spectral region (as usual, strongly coupled with $\nu \mathrm{C}-\mathrm{O}$ [28-32], and also with $\left.\omega \mathrm{CH}_{2}\right)$. As previously referred, $\nu \mathrm{C}-\mathrm{O}$ in neutral glycine also gives rise to a band in this region $\left(1100 \mathrm{~cm}^{-1}\right)$, which is easily identified by taking into consideration its high intensity (see Table 3 and Fig. 3).

Finally, the $\nu \mathrm{CC}$ and $\nu \mathrm{CN}$ modes also give rise to bands in this region and are predicted by the calculations to be very sensitive to the nature of the neighbouring groups. Interestingly, these stretching vibrations seem to couple extensively only in the acid salt. Calculated frequencies and intensities are 
in general good agreement with the experimental infrared and Raman data.

\subsection{Region below $800 \mathrm{~cm}^{-1}$}

The deformation modes involving the skeletal atoms $(\delta \mathrm{COO}, \omega \mathrm{COO}, \delta \mathrm{CC}=\mathrm{O}$ and $\delta \mathrm{NCC})$ appear in this spectral region, along with the torsional vibrations. The frequencies predicted for these vibrations (in particular $\tau \mathrm{C}-\mathrm{O}$ and $\omega \mathrm{COO}$ ) in the acid salt, on the basis of the dimer 'model' calculation, are in much better agreement with experimental data than those calculated using the monomer. The appearance of the band at $135 \mathrm{~cm}^{-1}$ in the Raman spectrum of this molecule, which was assigned to the $\delta \mathrm{C}-\mathrm{O} \ldots \mathrm{H}$ 'ring' mode, is also worth noting. This could not be interpreted on the basis of the monomer 'model' calculation.

The predicted frequency for the NCC bending mode in the acid salt is $269 \mathrm{~cm}^{-1}$ (for both $B_{\mathrm{u}}$ and $A_{\mathrm{g}}$ modes). Thus, we assigned the Raman band at $296 \mathrm{~cm}^{-1}$ to this mode. In the zwitterionic glycine, this vibration is predicted to occur at a higher frequency than in the acid salt (calculated value: 320 $\mathrm{cm}^{-1}$ ) and is here assigned to the Raman band at $364 \mathrm{~cm}^{-1}$, whose infrared counterpart occurs at 358 $\mathrm{cm}^{-1}$ [9]. The $\tau \mathrm{C}-\mathrm{N}$ mode (calculated value: 206 $\mathrm{cm}^{-1}$ ) was previously assigned to the infrared band at ca. $520 \mathrm{~cm}^{-1}$ (Raman: $509 \mathrm{~cm}^{-1}$ ), on the basis of neutron diffraction studies. Hence, it can be concluded that intermolecular interactions within the crystal significantly affect the frequency of this vibration relative to the value obtained for the simple isolated molecule 'model' used here. It should be pointed out that the failure of theoretical predictions using simple isolated molecule 'models' for torsional vibrations in crystals, has already been indicated for other molecules [32] and should be taken into consideration using this kind of approach.

\section{General conclusions}

Following from extensive comparisons between theoretical predictions and spectroscopic data (FT-IR, Raman and MI-IR) for neutral and zwitterionic forms of glycine, the ab initio theoretical vibrational calculations here presented for the acid and alkaline salts of glycine, using a moderate basis set $\left(6-31 \mathrm{G}^{*}\right)$ at the Hartree-Fock level of theory, produce helpful predictions of vibrational frequencies for the assignment of the vibrational spectra of these molecules. In addition, although generally not as accurate as the frequencies, the theoretically predicted intensities (both in infrared and in Raman) have proved useful in assignment of the spectra. Finally, a dimeric structure of the acid salt of glycine in the crystalline state was found to give best results in the prediction of the vibrational spectra of this compound.

\section{Acknowledgements}

The authors would like to thank Professor Yuri P. Blagoi, Dr. Eugeni D. Radchenko and Dr. Igor D. Reva (Institute for Low Temperature Physics and Engineering of the Ukrainian Academy of Sciences, Kharkov) for making available the MI-IR experimental facilities used in this work. R.F. also aknowledges Professor Hugh D. Burrows for his comments on this work. This study has been carried out under the PRAXIS XXI research programme (contract PRAXIS $/ 2 / 2.1 /$ QUI/412/94) also supported by FEDER.

\section{References}

[1] J.H. Caldwell, N.W. Daw, H.J. Wyatt, J. Physiol. 276 (1978) 277.

[2] S. Suzuki, T. Shimanouchi, M. Tsuboi, Spectrochim. Acta 19A (1963) 1195.

[3] S.F.A. Kettle, E. Lugwisha, J. Eckert, N.K. McGuire, Spectrochim. Acta 45A (1989) 533.

[4] R.W. Williams, V.F. Kalasinsky, A.H. Lowrey, J. Mol. Struct. 281 (1993) 157.

[5] B. Depuy, C. Garrigou-Lagrange, J. Chim. Phys. 65 (1968) 450.

[6] C. Destrade, C. Garrigou-Lagrange, M.-T. Forel, J. Mol. Struct. 10 (1971) 203.

[7] T.C. Cheam, J. Mol. Struct. 274 (1972) 289

[8] Y. Ding, K. Krogh-Jesperssen, J. Comput. Chem. 17 (1996) 338.

[9] K. Furic, V. Mohacek, M. Bonifacic, I. Štefanic, J. Mol. Struct. 267 (1992) 39.

[10] E.D. Radchenko, G.G. Sheina, N.A. Smorygo, Yu.P. Blagoi, J. Mol. Struct. 116 (1984) 386.

[11] A. Vijay, D.N. Sathyanarayana, J. Phys. Chem. 96 (1992) 10735. 
[12] A.H. Lowrey, V.F. Kalasinsky, R.W. Williams, Struct. Chem. 4 (1993) 289.

[13] Y. Ding, K. Krogh-Jesperssen, Chem. Phys. Lett. 199 (1992) 261.

[14] P.-G. Jönsson, ̊․ Kvick, Acta Cryst. 28B (1972) 1827.

[15] M.J. Frisch, G.W. Trucks, H.B. Schlegel, P.M.W. Gill, B.G. Johnson, M.W. Wong, J.B. Foresman, M.A. Robb, M. Head-Gordon, E.S. Replogle, R. Gomperts, J.L. Andres, K. Raghavachari, J.S. Binkley, C. Gonzalez, R.L. Martin, D.J. Fox, D.J. Defrees, J. Baker, J.J.P. Stewart, J.A. Pople, Gaussian 92/DFT, Revision G.3, Gaussian, Pittsburgh, PA, 1993.

[16] W.J. Hehre, R. Ditechefield, J.A. Pople, J. Chem. Phys. 56 (1972) 2257.

[17] H.B. Schlegel, Ph.D. Thesis, Queen's University, Kingston, Ontario, Canada, 1975.

[18] R. Fausto, TRANSFORMER (version 2.0), Departamento de Química, Universidade de Coimbra, Portugal, 1997.

[19] M.D.G. Faria, R. Fausto, BUILD-G (version 2.0) and VIBRAT (version 2.0), Departamento de Química, Universidade de Coimbra, Portugal, 1997.

[20] H. Fuher, V.B. Kartha, K.G. Kidd, P.J. Krueger, H.H. Mantsch, Natl. Res. Council Can. Bull. 15 (1976) 1.

[21] J.R. Durig, Invited Oral Presentation in the NATO-ASI
Conference on Low Temperature Molecular Spectroscopy, Ponta Delgada, Portugal, 1995.

[22] H. Susi, D.M. Byler, J. Mol. Struct. 63 (1980) 1.

[23] S. Bratoz, D. Hadzi, N. Sheppard, Spectrochim. Acta 8A (1956) 249.

[24] R.K. Khanna, M. Horak, E.R. Lippincott, Spectrochim. Acta 22A (1966) 1759.

[25] R.S. Krishnan, K. Krishnan, Proc. Indian Acad. Sci. 60 (1964) 11.

[26] N.D. Sokolov, Ann. Chim. 10 (1965) 497.

[27] A. Kulbida, R. Fausto, J. Chem. Soc. Faraday Trans. 89 (1993) 4257.

[28] A. Kulbida, M.N. Ramos, M. Rasanen, J. Nieminen, O. Schrems, R. Fausto, J. Chem. Soc. Faraday Trans. 91 (1995) 1571.

[29] R. Fausto, A. Kulbida, O. Schrems, J. Chem. Soc. Faraday Trans. 91 (1995) 3755.

[30] R. Fausto, P.J. Tonge, P.R. Carey, J. Chem. Soc. Faraday Trans. 90 (1994) 3491.

[31] R. Fausto, J. Mol. Struct. 377 (1996) 181.

[32] R. Fausto, Ph.D. Thesis, Departamento de Química, Universidade de Coimbra, Portugal, 1988. 